\title{
Structural causes of singlet/triplet preferences of Norrish Type II reactions in carbonyls
}

\author{
Keiran N. Rowell, ${ }^{\dagger}$ Scott H. Kable, ${ }^{\dagger}$ and Meredith J. T. Jordan*, \\ $\dagger$ School of Chemistry, University of New South Wales, Sydney \\ $\ddagger$ School of Chemistry, University of Sydney, Sydney \\ E-mail: meredith.jordan@sydney.edu.au
}

Phone: +6129351 4420 . Fax: +61293513329

\begin{abstract}
Photolysis thresholds are calculated for the Norrish Type II (NTII) intramolecular $\gamma$-hydrogen abstraction reaction in 22 structurally informative carbonyl species. The B2GP-PLYP excited state $S_{1}$ and $T_{1}$ thresholds agree well with triplet quenching experiments. However, many linear-response methods deliver poor $S_{1}$ energetics, which is explained by a $S_{1} / S_{0}$ conical intersection in close proximity to the $S_{1}$ transition state. Multiconfigurational CASSCF calculations confirm a conical intersection features across all carbonyl classes.

Structure-activity relationships are determined that could be used in atmospheric carbonyl photochemsitry modelling. This is exemplified for butanal, whose NTII quantum yields are too low when used as a 'surrogate' for larger carbonyls, since butanal lacks the $\gamma$-substitution that stabilises the 1,4biradical. Reaction on $T_{1}$ dominates only in species where the $S_{1}$ thresholds are high - typically ketones. The $\alpha, \beta$ unsaturated carbonyls cannot cleave the $\alpha-\beta$ bond, causing them to photoisomerise. A concerted $S_{0}$ NTII mechanism is calculated to be viable and may explain the recent detection of NTII photoproducts in the photolysis of pentan-2-one below the $T_{1}$ threshold.
\end{abstract}

\section{Introduction}

\section{The Norrish Type II reaction mech- anism}

The Norrish Type II (NTII) photolysis reaction is available in larger carbonyl species that have a $\gamma$-hydrogen. As shown schematically in Figure 1, the NTII reaction involves intramolecular $\mathrm{H}$-abstraction by a photoexcited $\mathrm{C}=\mathrm{O}$ chromophore to form a biradical. ${ }^{1-3}$ The NTII reaction dominates the photolysis of carbonyls with main alkyl chains of four carbon atoms or longer. ${ }^{4,5}$ NTII reactions can involve both the excited electronic singlet $\left(S_{1}\right)$ and triplet $\left(T_{1}\right)$ states as shown in Figure 1 below. The propensity for singlet or triplet reactions depends on the particular carbonyl species, ${ }^{6,7}$ and is dictated by the $S_{1}$ and $T_{1}$ NTII photolysis threshold for each carbonyl. In principle a similar $S_{0}$ pathway could also exist, and is examined in this paper.

With reference to Figure 1, if the available photon energy is above the photolysis threshold for the $S_{1}$ cyclic transition state (TS) then the 1,5-H-shift can occur on $S_{1}$, leading to a singlet biradical. ${ }^{8}$ However, if the photon energy is near or below the $S_{1}$ NTII threshold then reaction on $S_{1}$ will be slow and intersystem crossing (ISC) to $T_{1}$ can occur, resulting in the formation of a triplet biradical. The triplet biradical is long lived because it correlates with excited state products, that is, either the alkene or enol NTII product is in an electronic excited 


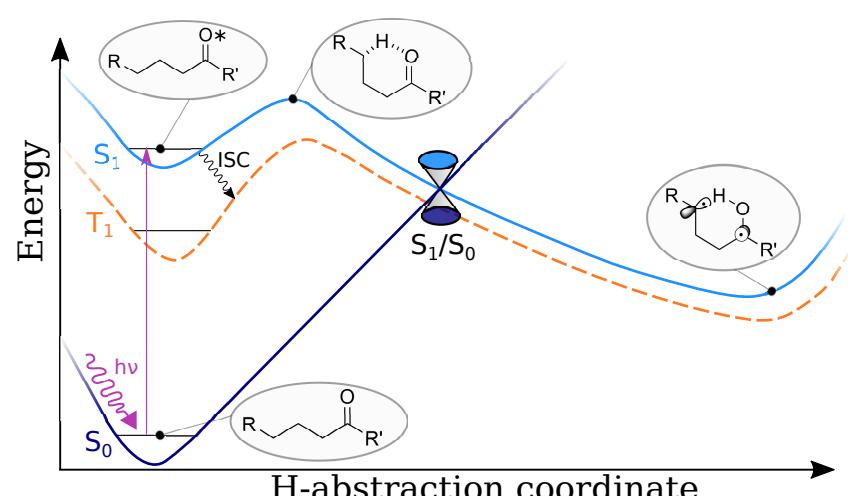

H-abstraction coordinate

Figure 1: Schematic illustrating the Norrish type II reaction pathways on $S_{1}$ (light blue) and $T_{1}$ (orange). Following photoexcitation to $S_{1}$ the carbonyl forms a cyclic 6-membered transition state and undergoes a 1,5$\mathrm{H}$-shift to form a 1,4-biradical. If the available energy is above the $S_{1}$ photolysis threshold a singlet biradical will be formed, otherwise intersystem crossing (ISC) to $T_{1}$ can occur, leading to a triplet biradical. A $S_{1} / S_{0}$ conical intersection is proposed to occur along the $S_{1}$ reaction pathway, allowing ultrafast crossing to $S_{0}$.

state, and these products are not accessible at actinic UV energies (> $300 \mathrm{~nm}$ ). The alkene and enol photoproducts observed in NTII reactions are ground state species, thus for NTII reaction to occur via $T_{1}$ there must be ISC to the $S_{0}$ state. A conical intersection (CI) between $S_{1}$ and $S_{0}$ is also proposed along the $S_{1}$ NTII reaction coordinate, ${ }^{9}$ lowering the lifetime of the singlet biradical by allowing ultrafast crossing to $S_{0}$ and hence rapid formation of NTII photoproducts. The different lifetimes of the singlet and triplet biradicals are also reflected in different NTII products observed for reaction on $S_{1}$ and $T_{1}$, such as cyclisation or retention of stereochemistry. ${ }^{10}$

The 1,4-biradical can undergo different subsequent reactions: either cleavage of the $\alpha, \beta$ bond to form an enol and an alkene (Scheme 1), or Norrish Yang cyclisation (NYC) to form a substituted cyclobutanol ${ }^{11,12}$ (Scheme 2). Additionally, reaction from the biradical can be frustrated, as the abstracted H-atom can backtransfer to re-form the original carbonyl.

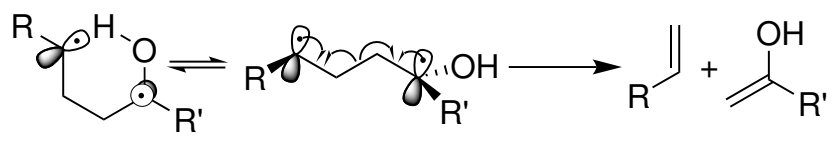

Scheme 1: Norrish Type II cleavage to an alkene and enol.

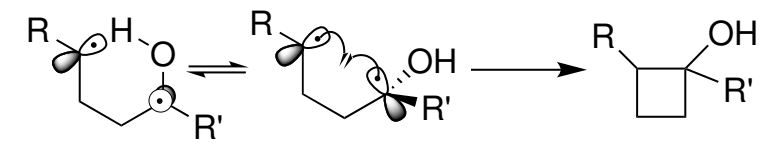

Scheme 2: Norrish Yang cyclisation to a substituted cyclobutanol.

The prevalence of the reactions shown in Schemes 1 and 2 depends on: the carbonyl substituents, ${ }^{10,13}$ chemical environment, ${ }^{14,15}$ and degree of energetic and conformational stabilisation of the biradical intermediate. ${ }^{1,3}$ Each of these factors influence the conformation of the $p$-type radical singly occupied molecular orbitals (SOMOs) of the biradical. The cleavage of the biradical to an alkene and an enol requires the SOMOs to overlap the sites of the newly forming $\mathrm{C}=\mathrm{C}$ bonds (Scheme 1 ), and these gauche conformations are readily accessible in carbonyls without bulky $\alpha$-substituents. ${ }^{10}$ Consequently, cleavage is the dominant NTII photolysis reaction in the gas phase for linear carbonyls.

Norrish Yang cyclisation occurs in many carbonyl species, ${ }^{10,12,16}$ and requires a conformation with in-phase overlap of the biradical SOMOs to form a new $\mathrm{C}-\mathrm{C}$ bond (Scheme 2). The NYC reaction is common for solvated carbonyls, ${ }^{15,16}$ and a synthetic strategy to form 4-membered rings is to use carbonyl substituents that promote conformations with overlapping SOMOs for cyclisation. ${ }^{17}$

However, NYC also occurs in the gas phase, provided a cyclisation conformation can be reached before cleavage to an alkene and enol occurs. Indeed, several classes of carbonyl species exclusively undergo NYC upon photolysis due to conformational effects. These include $\alpha$-diketones, ${ }^{18,19} \alpha$-fluoro ketones, ${ }^{20}$ and $\beta, \gamma$ unsaturated ketones. ${ }^{21}$ Additionally, intramolecular abstraction of hydrogens further than the $\gamma$-position can occur, ${ }^{22,23}$ but quantum yields (QYs) for these alternative abstractions are low whenever a $\gamma-\mathrm{H}$ is available ${ }^{24}$ and these alternate abstractions must result in cyclisation rather than cleavage. ${ }^{25}$ The canonical 6 membered NTII TS to form a 1,4-biradical is the most energetically favourable intramolecular $\mathrm{H}$-abstraction pathway due to its low degree of ring strain, ${ }^{17,26}$ and so is the only TS discussed in this paper. 
This paper is concerned with the NTII reaction in gas-phase carbonyls that bear the simple hydrocarbon chains typical of the volatile organic compounds (VOCs) found in high concentrations in the troposphere. ${ }^{27,28}$ Under tropospheric conditions NTII predominantly leads to alkene and enol photoproducts. ${ }^{29}$ Indeed, these are the only NTII photoproducts considered in the Master Chemical Mechanism. ${ }^{30,31}$ However, NYC is still observed as a minor channel in gas-phase photolysis of these 'typical' carbonyls, ${ }^{11,32}$ and the occurrence of a cyclisation product provides mechanistic insight.

In recent gas-phase FT-IR photolysis experiments on pentan-2-one, Kharazmi observed 1methylcyclobutanol as a photoproduct. ${ }^{32}$ For this to occur the biradical must be able to undergo internal bond rotation to access the conformation shown in Scheme 2, which does not happen with singlet biradicals as they retain stereochemistry in their photoproducts. ${ }^{8}$ Additionally, triplet quenching studies show that NYC photoproducts arise purely from triplet NTII reactions, since the $T_{1}$ biradical can undergo conformational rearrangement before cleavage occurs. ${ }^{12,33}$

This $T_{1}$ biradical cyclisation mechanism is consistent with the lifetime of the biradical measured in femtosecond mass spectrometry experiments on a series of ketones: the 1,5-H-shift occurs on a 70-90 fs timescale, while subsequent cleavage from this biradical is an order of magnitude slower at 400-700 fs. ${ }^{34}$

The photolysis data by Kharazmi on 1methylcyclobutanol formation from pentan-2one are also consistent with a triplet cyclisation mechanism. The QY of the NYC product of pentan-2-one is $\sim 0.05$ at $300 \mathrm{~nm}$ (above the expected $S_{1}$ NTII threshold) and increases for longer wavelengths, up to $\sim 0.17$ at $325 \mathrm{~nm} .{ }^{32}$ This suggests that NYC becomes increasingly important at lower photolysis energies since reaction on $S_{1}$ is no longer rapid, allowing ISC and reaction on $T_{1}$ to occur. The QY of 1 methylcyclobutanol then drops below 0.05 at $330 \mathrm{~nm}$, indicating that the $T_{1}$ NTII photolysis threshold also becomes inaccessible at this lower energy.

In order to understand the photolysis of larger carbonyls, it is therefore crucial to have accurate estimates of $S_{1}$ and $T_{1}$ NTII thresholds. The cause of the short $S_{1}$ biradical lifetimes, and the high rate of $\mathrm{H}$-backtransfer reversion to the starting carbonyl, was not well understood mechanistically when data was obtained in the 1970s. ${ }^{35}$ To explain these phenomena, the presence of a $S_{1} / S_{0}$ conical intersection (CI) along the NTII reaction pathway was proposed in the 1990s. ${ }^{9,36}$ The possibility of a concerted $S_{0}$ NTII photolysis pathway has also been proposed, ${ }^{16}$ and recent wavelength-dependent FT-IR photolysis data shows the formation of NTII photoproducts in pentan-2-one at energies below the predicted $T_{1}$ NTII threshold. ${ }^{32}$

\section{Dataset of NTII-capable carbonyls}

This paper aims to derive NTII threshold structure-activity relationships (SARs) generalisable to a large variety of carbonyl species. A significant proportion of biogenic volatile organic compounds (VOCs) are larger (5-6 carbon) molecules. ${ }^{28,37-39}$ This includes the atmospherically abundant isoprene-derived C5 carbonyls, ${ }^{40}$ and the C6 carbonyls like 'leaf-aldehyde' (hex3-enal). ${ }^{41}$

Carbonyls larger than C6 are not studied here because: the volatility and abundance of carbonyls decrease with chain length; ${ }^{28}$ substitution patterns adjacent to incipient $\gamma-\mathrm{C}^{\bullet}$ and $\mathrm{C}^{\bullet}-\mathrm{O}$ radical sites can still be studied with molecules of this size; and the computational expense when performing $\mathcal{O}\left(N^{7}\right)$ (EOM-)CCSD calculations is limited.

The 22 NTII-capable carbonyl species studied here are shown in Scheme 3, and their systematic names are listed below by carbonyl class:

- Saturated aldehydes: butanal, pentanal, 2-methylpentanal, 3-methylpentanal, 4-methylpentanal, 2-ethylbutanal, 3,3dimethylbutanal, hexanal, hex-3-enal;

- Saturated ketones: pentan-2-one, hexan-2-one; 3-methylpentan-2-one, 4methylpentan-2-one;

- $\alpha, \beta$-Unsaturated aldehydes: but-2enal, 2-methylpent-2-enal, 3-methylpent- 


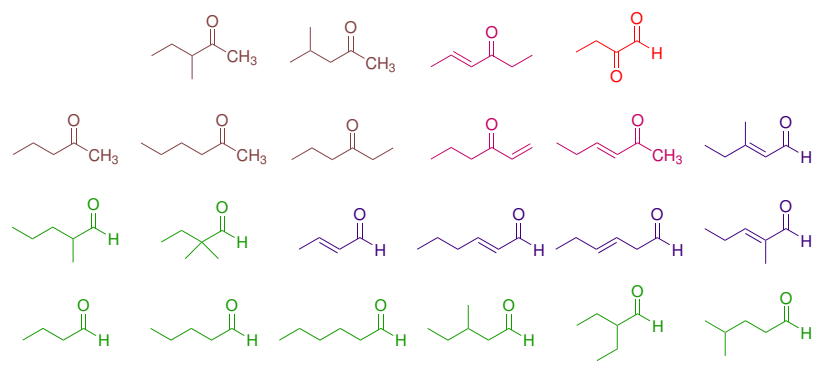

Scheme 3: Dataset of carbonyl bearing $\gamma$-hydrogens, for which Norrish Type II reaction thresholds are calculated.

2-enal, hex-2-enal;

- $\alpha, \beta$-Unsaturated ketones: hex-3-en-2one, hex-1-en-3-one, hex-4-en-3-one;

- Dicarbonyls: 2-oxobutanal.

\section{Previous electronic structure calcu- lations of the NTII reaction}

This paper is only concerned with NTII reaction energetics to determine photolysis SARs. Theoretical work on other mechanistic aspects of the NTII reaction, such as the dynamics of the biradical, are available in other studies, but only for a few carbonyl species. ${ }^{42-44}$ An overview of the previous modelling work for the NTII reaction in carbonyls is presented below to give context to this current study.

Early electronic structure calculations Early MINDO/3 semi-empirical calculations in 1978 were able to provide the key mechanistic details of the $S_{1}$ and $T_{1}$ NTII reactions, including the importance of the biradical intermediate, ${ }^{45}$ though the method mistakenly identifies the $S_{1} / S_{0}$ region of degeneracy on the path to the biradical formation as an avoided crossing, rather than a true conical intersection. Later modelling on $T_{1}$ using UHF and UMP2 methods made clear the preference for NTII $\gamma-\mathrm{H}$ abstraction via a planar 6-membered TS, and its similarity to the ground state retro-ene and McLafferty rearrangements, ${ }^{46}$ which will be discussed later in the context of an $S_{0}$ NTII mechanism. Semi-empirical methods have also been used to study other mechanistic steps involving the 1,4-biradical, including cyclisation and H-backtransfer pathways. ${ }^{47}$

Electronic states of NTII in unsaturated carbonyls The photochemistry of butyrophenone has been modelled using multiconfigurational complete active space (CAS) methods. ${ }^{48}$ Butyrophenone has a phenyl group conjugated with the $\mathrm{C}=\mathrm{O}$ moiety and as an aromatic carbonyls it has similarities to the $\alpha, \beta$-unsaturated carbonyls discussed here. This includes a $T_{2}$ state that is energetically accessible at tropospheric photon energies, ${ }^{49}$ as well as a $S_{1} / T_{2} / T_{1}$ three-state intersection. This intersection is not at configurations accessed by the triplet biradical, and does not intersect with $S_{0}$, and so does not decrease the triplet biradical lifetime. However, an $S_{1} / S_{0}$ CI was located. From this, it can be surmised that only the $T_{1}$ NTII reaction results in a biradical with significant lifetime, even in species where conjugation to the $\mathrm{C}=\mathrm{O}$ moiety complicates the electronic states involved.

\section{NTII/NTI $a$ competition and substituent} effects Both the Norrish Type I $\alpha$-bond cleavage (NTI $a$ ) and NTII reactions in butanal have been modelled using the CASSCF, DFT, and MP2 levels of theory by Chen and Fang. ${ }^{50}$ These calculations demonstrate that in the tropospheric energy range, NTI $a$ reaction in butanal only occurs on $T_{1}$ after ISC; whereas NTII can occur on both the $S_{1}$ or $T_{1}$ states. Chen and Fang also located an $S_{1} / S_{0}$ CI along the $S_{1}$ NTII reaction coordinate, after the $S_{1} 1,5-\mathrm{H}$-shift TS. A recent theoretical study highlights the role of 'singlet state only' NTII and NTI $a$ mechanisms in 5-methylhexan-2-one derivatives. ${ }^{51}$ While this study also calculates reaction enthalpies on substituted reaction products, this is estimated with single point energy calculations on products and not by locating TSs, and so does not provide data on photolysis thresholds.

Dynamics of the biradical The dynamics of the triplet 1,4-biradical formed from pentanal have been simulated using semi-empirical multireference configuration interaction (MRCI) cal- 
culations at two timescales: 10 ps and 100 ps. $^{43}$ In the 10 ps simulations both NTI $a$ and the NTII $\gamma-\mathrm{H}$ transfer occurred. In the 100 ps simulations neither the biradical cyclisation nor cleavage were observed, inconsistent with the 400-700 fs biradical lifetime estimated by femtosecond mass-spectrometry experiments. ${ }^{34}$ Nevertheless, these dynamics simulations indicate that the 1,5$\mathrm{H}$-shift and subsequent reactions occur on separate time-scales in the triplet biradical, allowing conformational rearrangements to occur. The MRCI dynamic simulations predicted a NTI $a$ QY of 0.34 and NTII QY of 0.66 on the 100 ps timescale, in qualitative but not quantitative agreement with observed NTII QYs of $\sim 0.8$ in pentanal. ${ }^{4,52}$

\section{Multiconfigurational calculations on pro-} totypical aldehydes Kletskii et al. used the multiconfigurational XMCQDPT2 method $^{53}$ to characterise Norrish reactions in butanal, pentanal, and but-2-enal. ${ }^{54}$ XMCQPDT2 provides a correct representation of wavefunctions with multireference character, and regions with electronic state degeneracies such as conical intersections, both of which are incorrectly modelled with linear response (LR) time-dependent density functional theory (TD-DFT) or equationof-motion coupled cluster (EOM-CC) methods. ${ }^{55}$ However, like CASPT2, the XMCQDPT2 method is more computationally demanding than single-reference methods, ${ }^{56}$ and the energetics delivered for carbonyl photochemistry can often be worse than those from many DFT functionals. ${ }^{26}$ While the Kletskii et al. paper provides the largest qualitatively correct survey of the electronic structure and energies along the NTII reaction for a few carbonyls (butanal, pentanal, pentan-2-one, but-2-enal), we wish here to expand the study of the NTII to many more more molecules.

\section{Computational Methods}

The computational protocols used in this paper to study $S_{1}, T_{1}$, and $S_{0}$ NTII photolysis thresholds are largely the same as reported by the authors in an earlier publication on the NTI reaction in carbonyls. ${ }^{49}$ Photolysis thresholds are calculated by the addition of the intrinsic reaction barrier (relative to the relevant electronic state minimum) to the $0-0$ excitation energy (from the $S_{0}$ minimum to the excited state minimum). The $0-0$ excitation energies used here are calculated using protocol detailed by the authors in an earlier paper studying carbonyl excitation energies. ${ }^{57}$ The methods are summarised below.

\section{$S_{0} \rightarrow S_{1}$ Excitation energies}

Briefly, the chosen protocol for 0-0 excitation energies is: EOM-CCSD vertical excitation energies scaled by factors derived from CCSDR(3) results, ${ }^{57}$ EOM-CCSD//TD-CAM-B3LYP $S_{1}$ relaxation energies, and (TD-)CAM-B3LYP zeropoint vibrational energies (ZPVEs). A moleculeindependent energy shift, equal to the mean signed error of the 0-0 excitation energy protocol, is applied to correct for the lack of triples in the calculated relaxation energy, as discussed in the earlier carbonyl excitation paper. ${ }^{57}$

\section{Optimisation of $S_{1} \mathbf{1}, 5-\mathbf{H}$-shift tran- sition states}

In our earlier study on the NTI reaction, photolysis thresholds and intrinsic reaction barriers determined using TD-B3LYP were found to reasonable agreement with respect to experimental data. ${ }^{49}$ Here, however, the presence of an $S_{1} / S_{0}$ CI near $S_{1}$ biradicals and/or TS structures means that linear response theories, like TD-DFT, may not be appropriate and multiconfigurational methods must be used. ${ }^{58}$ This, and other potential limitations of the theoretical methods used, are discussed below.

Hydrogen-transfer TSs can be difficult to locate with DFT methods, and usually require functionals with a high component of Fock exchange. ${ }^{59,60}$ Notably, B3LYP is poor at describing hydrogen transfer $\mathrm{TSs}^{61,62}$ and neither TDB3LYP calculations nor TD-BH\&HLYP calculations (with $50 \% \mathrm{HF}$ ) could locate the $S_{1} 1,5-$ H-shift TSs here. Instead, TD-CAM-B3LYP is used to successfully optimise the required $S_{1}$ 
TSs, noting that, from earlier work, ${ }^{49,57}$ the TDB3LYP and TD-CAM-B3LYP $S_{1}$ results had comparable accuracy. Single point energies were determined on $S_{1}$ at the TD-B2GP-PLYP level of theory using TD-CAM-B3LYP geometries.

A zero-point vibrational energy (ZPVE) scaling factor of 0.976 was used for $S_{1}$ TD-CAMB3LYP/def2-TZVP ZPVE corrections. This value was derived for CAM-B3LYP/ma-TZVP calculations, but it has been shown that the minimal augmentation has negligible effect on the scale factor. ${ }^{63,64}$ It is also assumed, in the absence of additional data, that the scale factor for $S_{0}$ ZPVEs is also applicable to $S_{1}$. As discussed previously, ${ }^{49}$ the few literature studies on excited state scaling factors ${ }^{65,66}$ indicate that this assumption will lead to differences of less than $1 \mathrm{~kJ} / \mathrm{mol}$ in ZPVE corrected energies.

The 1,5-H-shift TS to formation of the 1,4biradical, shown in Figure 1, is also likely to have significant biradical character. The $S_{1} / S_{0}$ CIs are expected to be near the reaction coordinate for formation of the 1,4-biradical, ${ }^{9,54}$ and again will have biradical character. In principle all of these configurations should be described using multiconfigurational methods. ${ }^{67,68}$ For $S_{0}$ biradicals, DFT functionals are able to deliver reasonable energies. ${ }^{69,70}$ For carbonyls specifically, previous $\mathrm{PhD}$ work by Shaw on carbonyl epoxidation reactions was able to predict $S_{0}$ biradical TSs accurately with UM06-2X calculations. ${ }^{26}$ This same work showed that carbonyl $T_{1}$ stationary points with significant biradical character calculated with UM06-2X level were within $10 \mathrm{~kJ} / \mathrm{mol}$ benchmark results from higher levels of theory. ${ }^{26}$

This work also studied carbonyl 1,5-H-shift TSs on $S_{1}$ using DFT and multiconfigurational methods, and while TD-M06-2X intrinsic barriers were $\sim 20 \mathrm{~kJ} / \mathrm{mol}$ higher than those calculated using the XMCQDPT2 method (see Table $\mathrm{S} 1$ ), the TD-DFT results appeared to be in better agreement with experiment. Thus, somewhat fortuitously, DFT-based methods appear capable of predicting $S_{1}$ NTII energies to somewhat acceptable accuracy — assuming TD-DFT formalism is valid, that is, the effects of the $S_{1} / S_{0}$ CI are negligible.

\section{CASSCF calculations}

Multiconfigurational CASSCF calculations were used to determine a qualitatively accurate picture of any $S_{1} / S_{0}$ minimum energy conical intersections (MECIs). Gaussian 09 or Gaussian 16 were used for all CASSCF calculations and MECI searches. TD-CAM-B3LYP $S_{1}$ NTII TS geometries were used as the starting points for all CASSCF MECI searches, since the $S_{1} / S_{0}$ MECIs were assumed to be in close proximity to the $S_{1} 1,5-\mathrm{H}$-shift TSs.

An $(8,7)$ active space was used for initial optimisations, matching previous literature. ${ }^{50,54,71}$ The four occupied orbitals in the active space are the: $\gamma \mathrm{C}-\mathrm{H} \sigma, \mathrm{C}-\mathrm{O} \sigma, \mathrm{C}=\mathrm{O} \pi$, and oxygen lone pair $n$ orbitals; and the virtual orbitals are the: $\gamma \mathrm{C}-\mathrm{H} \sigma^{*}, \mathrm{C}-\mathrm{O} \sigma^{*}$, and $\mathrm{C}=\mathrm{O} \pi^{*}$ orbitals. This was then expanded to a $(10,8)$ active space by including the other oxygen-centred non-bonding orbital (see Figure S1). For unsaturated species the $\pi$ and $\pi^{*}$ orbitals at the point of unsaturation were also included in the active space, and in these species the $\mathrm{C}-\mathrm{O} \sigma$ and $\sigma^{*}$ orbitals were removed to keep a manageable $(10,8)$ active space (see Figure S2). The MECI structures calculated here with the $6-31+\mathrm{G}(\mathrm{d})$ basis set for butanal, pentanal, pentan-2-one, and but-2-enal were consistent with those calculated by Kletskii et al. with the larger $6-311++\mathrm{G}(\mathrm{d}, \mathrm{p})$ basis set. ${ }^{54}$ Further details are provided in the supporting information.

\section{Results}

\section{Norrish Type II reaction energies}

Our best calculations for: $S_{1}, T_{1}$, and $S_{0}$ NTII photolysis thresholds relative to the $S_{0}$ minimum; intrinsic reaction barriers relative to the corresponding $S_{1}$ or $T_{1}$ minimum; and $S_{0} \rightarrow T_{1}$ and $S_{0} \rightarrow S_{1}$ 0-0 excitation energies; are reported in Table 1. All energies in Table 1 are ZPVE corrected.

To the author's knowledge, no direct experimental determination of photolysis threshold energies for the NTII reaction in any carbonyl has been published. An indication of the $S_{1}$ and $T_{1}$ photolysis thresholds can be inferred from 
Table 1: 0-0 excitation energies, NTII photolysis thresholds, and NTII intrinsic reaction barriers, as indicated. $(\mathrm{kJ} / \mathrm{mol})$

\begin{tabular}{|c|c|c|c|c|c|c|c|}
\hline & \multicolumn{2}{|c|}{ 0-0 Excitation Energy } & \multicolumn{3}{|c|}{ Photolysis Threshold } & \multicolumn{2}{|c|}{ Intrinsic Barrier } \\
\hline & \multirow{2}{*}{$\begin{array}{c}S_{0} \rightarrow S_{1} \\
\text { Composite }^{a}\end{array}$} & \multirow{2}{*}{$\begin{array}{c}S_{0} \rightarrow T_{1} \\
\text { B2GP-PLYP }\end{array}$} & \multirow{2}{*}{$\frac{S_{1}}{\text { Composite }}$} & \multirow{2}{*}{$\begin{array}{l}T_{1} \\
\mathrm{~B} 2 \mathrm{GH}\end{array}$} & \multirow{2}{*}{$\begin{array}{l}S_{0} \\
\text { PLYP }\end{array}$} & \multirow{2}{*}{$\begin{array}{c}S_{1} \\
\text { B2GP-PLYP// } \\
\text { CAM-B3LYP }\end{array}$} & \multirow{2}{*}{$\begin{array}{c}T_{1} \\
\text { B2GP-PLYP }\end{array}$} \\
\hline & & & & & & & \\
\hline Aldehydes: & & & & & & & \\
\hline Butanal & 353 & 325 & $382(\sim 382)^{b}$ & 368 & 239 & 29 & 43 \\
\hline Pentanal & 352 & 324 & 369 & 359 & 236 & 17 & 35 \\
\hline Hexanal & 354 & 320 & 374 & 358 & 232 & 20 & 39 \\
\hline 2-Ethylbutanal & 362 & 324 & 376 & 359 & 225 & 14 & 35 \\
\hline 2-Methylpentanal & 348 & 323 & 359 & 355 & 227 & 11 & 32 \\
\hline 3-Methylpentanal & 350 & 321 & 367 & 358 & 225 & 17 & 37 \\
\hline 4-Methylpentanal & 352 & 324 & 362 & 351 & 233 & 10 & 27 \\
\hline 3-3-Dimethylbutanal & 346 & 318 & 372 & 364 & 224 & 26 & 46 \\
\hline $\begin{array}{l}\text { Hex-3-enal } \\
\text { Ketones: }\end{array}$ & 349 & 320 & 387 & 376 & 280 & 38 & 56 \\
\hline Pentan-2-one & 371 & 339 & $399(>382)^{b}$ & 381 & 242 & 28 & 42 \\
\hline Hexan-2-one & 366 & 336 & $381(<382)^{b}$ & 370 & 239 & 15 & 34 \\
\hline 3-Methylpentan-2-one & 358 & 333 & 379 & 374 & 231 & 21 & 42 \\
\hline 4-Methylpentan-2-one & 362 & 335 & 387 & 382 & 239 & 25 & 46 \\
\hline $\begin{array}{l}\text { Hexan-3-one } \\
\text { Enals: }\end{array}$ & 368 & 338 & 394 & 383 & 243 & 26 & 45 \\
\hline But-2-enal & 330 & 298 & 335 & 323 & - & 5 & 25 \\
\hline Hex-2-enal & 327 & 298 & 318 & 314 & - & -9 & 16 \\
\hline 2-Methylpent-2-enal ${ }^{c}$ & 335 & $247^{c}$ & 323 & 321 & - & -12 & $74^{c}$ \\
\hline $\begin{array}{l}\text { 3-Methylpent-2-enal } \\
\text { Enones: }\end{array}$ & 320 & 291 & 311 & 308 & - & -9 & 17 \\
\hline Hex-3-en-2-one & 315 & 296 & 319 & 316 & - & 4 & 20 \\
\hline Hex-1-en-3-one & 313 & 288 & 341 & 332 & 229 & 28 & 44 \\
\hline $\begin{array}{l}\text { Hex-4-en-3-one } \\
\text { Dicarbonyls: }\end{array}$ & 324 & 298 & 341 & 330 & - & 17 & 32 \\
\hline 2-Oxobutanal & 251 & 125 & 297 & 148 & 128 & 46 & 23 \\
\hline
\end{tabular}

${ }^{a}$ Scaled EOM-CCSD//B2GP-PLYP $E^{\text {vert }}+$ EOM-CCSD//TD-CAM-B3LYP $E^{\text {relax }}+$ (TD-)CAM-B3LYP $\Delta E^{\text {ZPVE }}+\Delta$ HLC, as described in reference 57 .

${ }^{b}$ Experimental photolysis threshold energy bounds are shown in parentheses, estimated from triplet quenching studies (see text).

${ }^{c}$ 2-Methylpent-2-enal has a low calculated $S_{0} \rightarrow T_{1} 0-0$ energy, and high $T_{1}$ NTII barrier, because the $S_{0}$ minimum and $T_{1}$ NTII TS are calculated to be planar, while the alkyl group in the $T_{1}$ minimum is calculated to be twisted around the $\mathrm{C}=\mathrm{C}$ bond.

triplet quenching studies of particular carbonyl species, or the formation of NTII photoproducts thought to arise only from $T_{1}$ NTII reactions (such as cyclobutanols). ${ }^{10}$ Experimental triplet quenching studies on butanal, pentan-2one, and hexan-2-one have been performed using a single photolysis wavelength of $313 \mathrm{~nm}(382$ $\mathrm{kJ} / \mathrm{mol}) .{ }^{6,7}$ For butanal both singlet and triplet NTII products were observed, indicating a $S_{1}$ NTII threshold of approximately $382 \mathrm{~kJ} / \mathrm{mol}$, since reaction on $S_{1}$ must be relatively slow for ISC to be competitive. ${ }^{7}$ Only triplet NTII products were observed in the $313 \mathrm{~nm}$ photolysis of pentan-2-one, indicating an $S_{1}$ NTII photolysis threshold above $382 \mathrm{~kJ} / \mathrm{mol}$, and a $T_{1}$ threshold below $382 \mathrm{~kJ} / \mathrm{mol}^{6}$ The NTII photoproducts for hexan-2-one at $313 \mathrm{~nm}$ were all unquenchable by triplet quenchers, i.e. the photoproducts were all singlets, and so the $S_{1}$ NTII threshold should be below $382 \mathrm{~kJ} / \mathrm{mol}^{6}$

The NTII $S_{1}$ and $T_{1}$ photolysis thresholds predictions in Table 1 are consistent with these experiments: the $S_{1}$ NTII threshold predicted for butanal $(382 \mathrm{~kJ} / \mathrm{mol})$ is close to $382 \mathrm{~kJ} / \mathrm{mol}$, while pentan-2-one is predicted to have a higher $S_{1}$ NTII threshold $(399 \mathrm{~kJ} / \mathrm{mol})$, and hexan2-one a slightly lower $S_{1}$ NTII threshold (381 $\mathrm{kJ} / \mathrm{mol}$ ). The predicted thresholds in Table 1 are also in quantitative agreement with TD-M06$2 \mathrm{X} / 6-311++\mathrm{G}(3 \mathrm{df}, 2 \mathrm{p})$ calculations by Shaw, which predict $S_{1}$ NTII photolysis thresholds of: $385 \mathrm{~kJ} / \mathrm{mol}$ for butanal, $368 \mathrm{~kJ} / \mathrm{mol}$ for pentanal, 
$398 \mathrm{~kJ} / \mathrm{mol}$ for pentan-2-one, and $379 \mathrm{~kJ} / \mathrm{mol}$ for hexan-2-one. ${ }^{26}$

More broadly, there is a lack of NTII photolysis data on the other carbonyl species in Table 1. However the predicted energies in Table 1 are consistent with some general features of NTII photolysis:

- The relative proportion of $S_{1}$ and $T_{1}$ NTII reaction products of saturated carbonyls is wavelength-dependent within the actinic range. ${ }^{32}$ This provides upper and lower bounds for the NTII photolysis thresholds of $\sim 300-330 \mathrm{~nm}(362.5-400 \mathrm{~kJ} / \mathrm{mol})$, which agrees with Table 1.

- Photolysis is competitive between the $S_{1}$ and $T_{1}$ states, implying that their photolysis thresholds must be similar in energy. Given that $S_{0} \rightarrow S_{1}$ 0-0 excitation energies are higher than $S_{0} \rightarrow T_{1}$ 0-0 excitation energies, the NTII intrinsic reaction barriers must be lower on $S_{1}$. In contrast, NTI $a$ intrinsic reaction barriers are 50-70 $\mathrm{kJ} /$ mol higher on $S_{1}$ than on $T_{1} \cdot{ }^{49}$

- Predicted NTII thresholds for $\alpha, \beta$ unsaturated carbonyls are lower than those for saturated species. On both $S_{1}$ and $T_{1}$, these thresholds are all lower than $340 \mathrm{~kJ} / \mathrm{mol}$. This is partly due to the lower $0-0$ excitation energies of $\alpha, \beta$-unsaturated carbonyls due to delocalisation of their frontier orbitals, ${ }^{49,57}$ but also low intrinsic barriers due to an allylic resonance stabilisation effect that will be discussed later.

The TD-B2GP-PLYP//TD-CAM-B3LYP $S_{1}$ NTII intrinsic reaction barriers in Table 1 are small, mostly under $30 \mathrm{~kJ} / \mathrm{mol}$. For some $\alpha, \beta$ unsaturated species they are negative, indicating a barrierless $S_{1}$ NTII reaction pathway. Below, these $S_{1}$ intrinsic reaction barriers are calculated using a range of single point energy methods, and issues in the calculation of $S_{1}$ NTII TS energies are identified and diagnosed.

\section{Benchmarking $S_{1}$ NTII intrinsic re- action barriers}

Three of the $S_{1}$ NTII intrinsic reaction barriers in Table 1 are negative, those for: hex-2-enal, 2-methylpent-2-enal, and 3-methylpent-2-enal. These reactions may indeed be barrierless, or the TD-B2GP-PLYP NTII TSs on $S_{1}$ may have a different geometry to those optimised with TDCAM-B3LYP. A third option is the presence of an $S_{1} / S_{0}$ CI may degrade the quality of the TD-DFT energies. There is therefore a need to benchmark the $S_{1}$ NTII intrinsic reaction barriers and characterise any CIs.

Unfortunately, there are no reliable experimental benchmarks for the $S_{1}$ NTII intrinsic reaction barriers; the ranges extracted from experiment are not sufficiently precise. There are a few previous literature calculations of these $S_{1}$ intrinsic reaction barriers, ${ }^{5,26,54}$ and these are included in Table S1, where they are compared to calculations with four linear response methods (TD-CAM-B3LYP, TD-M06-2X, TDB2GP-PLYP and EOM-CCSD) on all carbonyl species in this paper. In short, large energetic discrepancies are seen between calculations using these different methods.

The multireference XMCQDPT2 calculations of Kletskii et al., which are based on CASSCF geometries, predicted lower barriers than the TD-M06-2X calculations of Shaw, as does the CIS(D) barrier predicted for butanal by Tadic et al. The TD-M06-2X/6-311++G(3df,2p) calculations by Shaw predicted $S_{1}$ NTII intrinsic reaction barriers approximately $10-20 \mathrm{~kJ} / \mathrm{mol}$ higher than CASSCF and CASPT2 values, ${ }^{26}$ however it was shown that these higher intrinsic TD-M06-2X barriers are more consistent with experimental photolysis thresholds. Importantly, from the comparisons in Table S1 predicted $S_{1}$ intrinsic reaction barriers are sensitive to the level of theory used.

Moreover, Kletskii et al. find that, at the XMCQDPT2 level of theory, the $S_{1}$ NTII reaction in but-2-enal is barrierless, that is, a negative energy is reported in Table S1. A number of $S_{1}$ NTII reactions, for enals and enones, are also found to be barrierless at the levels of theory used here. Literature calculations on carbonyl $S_{1}$ 
NTII intrinsic reaction barriers using wavefunction methods have predicted low $(<15 \mathrm{~kJ} / \mathrm{mol})$ barriers, ${ }^{5,54}$ or even barrierless processes, for $\alpha, \beta$-unsaturated species like but-2-enal. ${ }^{54}$

A possible reason for the large energetic discrepancies between methods could be the breakdown of the linear response approximation at either, or both, the $S_{1}$ minimum energy geometry and the $S_{1}$ NTII TS. Single point energies at $S_{1}$ minimum energy geometries are reported in Table S2. These are broadly consistent for all four levels of theory, and moreover are consistent with experimental 0-0 excitation energies. Therefore, the discrepancies arise from calculations on the $S_{1}$ NTII TS, and this is shown by anomalously low $S_{0} \rightarrow S_{1}$ single point energies at $S_{1}$ NTII TS geometries reported in Table S3.

\section{Comparison between $S_{1}$ and $T_{1}$ B2GP- PLYP energies}

These large variations and/or oscillations in energy are diagnostic of the presence of a MECI near the TS. ${ }^{55,72-74}$ As an additional 'flag' for the presence of an $S_{1} / S_{0}$ CI, the $S_{1}$ NTII intrinsic reaction barriers were compared to the corresponding $T_{1}$ energies. For both $S_{1}$ and $T_{1}$, the NTII reaction takes place via a species with biradical character where the radical centres are separated by two carbon atoms. As a result, the electronic character of the NTII TSs should be similar on both electronic states, and should display similar energetic trends with respect to substitution for the saturated carbonyls considered. This is not necessarily true for the enals and enones, however, as the double-bond lies between the radical centres in the $\alpha, \beta$-unsaturated species in this dataset. Furthermore, unlike the $S_{1}$ energies, the optimised $T_{1}$ TS energies are expected to be considerably more reliable because the $T_{2}$ state is significantly higher in energy, and a $T_{2} / T_{1} \mathrm{CI}$ is not expected. The $T_{1}$ UB2GP-PLYP calculations are expected to yield accurate and reliable energies as they have been validated against experiment. ${ }^{49}$ Our calculated NTII intrinsic reaction barriers on $S_{1}$ (open symbols) and $T_{1}$ (closed symbols) are compared in Figure 2 below.

The $S_{1}$ TD-B2GP-PLYP//TD-CAM-B3LYP energies in Figure 2 follow, for the most part, the same energetic trends as the $T_{1}$ UB2GPPLYP energies. They are systematically 15-30 $\mathrm{kJ} / \mathrm{mol}$ lower than the corresponding $T_{1}$ energies. However, the trends in NTII intrinsic reaction barriers with substitution patterns are not calculated to be in quantitative agreement for the ketones on $S_{1}$ and $T_{1}$. The intrinsic $T_{1}$ NTII barrier is much higher than the $S_{1}$ barrier because, as in the footnote of Table 1 , the $T_{1}$ minimum energy geometry of this molecule is calculated to be twisted around the $\mathrm{C}=\mathrm{C}$ bond, whereas the $S_{0}$ and $S_{1}$ minima are planar. Additionally, the $S_{1}$ NTII intrinsic reaction barrier for 2-oxobutanal is higher than the $T_{1}$ energy, but this may be due to the different electronic nature of $\alpha$-dicarbonyls. ${ }^{75,76}$ Thus, comparison of the $S_{1}$ TD-B2GP-PLYP and $T_{1}$ UB2GP-PLYP energies in Figure 2 does not indicate, in itself, a failure of the TD-DFT method.

In contrast, the EOM-CCSD//TD-CAMB3LYP $S_{1}$ NTII intrinsic reaction barriers shown in Figure 2 (open markers with internal crosses) are inconsistent with the $T_{1}$ energies. The butanal, 3-methylpentanal, 3,3-dimethylbutanal, hexan-2-one, hexan-3-one, hex-1-en-3-one, and 2-oxobutanal EOM-CCSD//TD-CAM-B3LYP $S_{1}$ intrinsic reaction barriers are higher than, or close to, the corresponding $T_{1}$ barrier. The EOM-CCSD $S_{1}$ energy for 2-oxobutanal, however, is similar to the TD-B2GP-PLYP $S_{1}$ energy. For the other six carbonyls, the discrepancy may be indicative of a breakdown of the linear response theory in the vicinity of a CI. The only caveat is that EOM-CC energies are extremely sensitive to geometry. ${ }^{77}$ To investigate this further, $S_{1} / S_{0}$ MECI searches were performed on select carbonyl species, and a range of diagnostics determined.

\section{Further diagnostics for $S_{1}$ calculations}

Diagnostic calculations were performed on the TD-CAM-B3LYP $S_{1}$ NTII TS geometries, as well as the $S_{1}$ minimum energy geometries. This includes the $\mathcal{T}_{1}$ CCSD diagnostic for multireference character, ${ }^{78,79}$ as well as $S_{1}-S_{0}$ and $S_{2}-S_{1}$ electronic state energy gaps. These diagnostic calculations are reported in Table S4. 


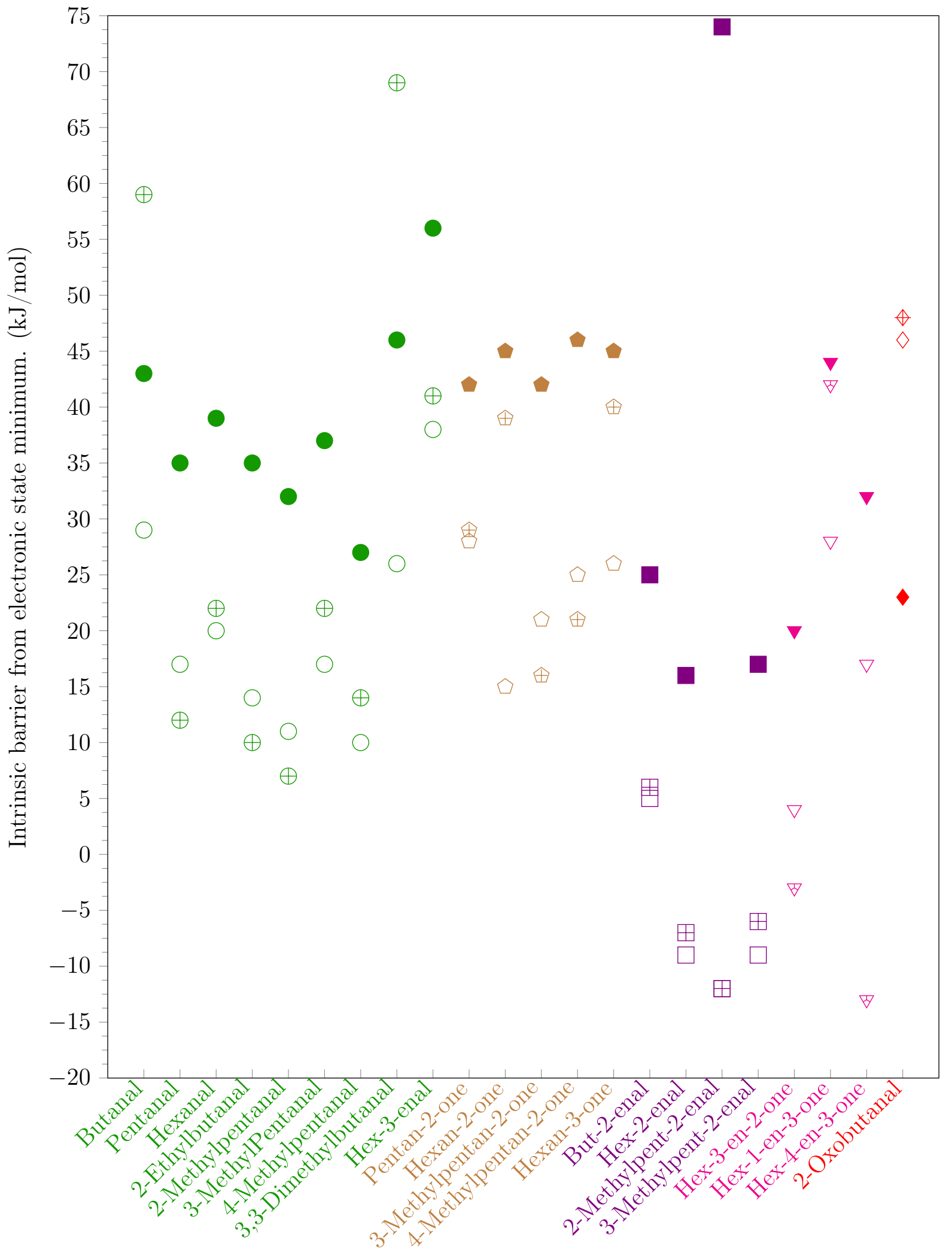

Figure 2: Intrinsic Norrish Type II reaction barriers, relative to relevant electronic state minimum for each molecule. Filled markers $(-)$ are $T_{1}$ intrinsic barriers, calculated at the UB2GP-PLYP/def2-TZVP level of theory. Open markers are $S_{1}$ barriers. Completely unfilled $(\bigcirc)$ markers show intrinsic $S_{1}$ barriers calculated at the TD-B2GP-PLYP/def2TZVP//TD-CAM-B3LYP/def2-TZVP level of theory, while open markers with internal crosses $(\oplus)$ show intrinsic $S_{1}$ barriers calculated at the EOM-CCSD/def2-TZVP//TD-CAM-B3LYP/def2-TZVP level of theory. All energies are ZPVE corrected. Symbol shape and colour indicate carbonyl classes: linear aldehydes $(\bullet)$, branched aldehydes $(\mathbf{\Lambda})$, linear ketones $(\bullet)$, enals $(\boldsymbol{\nabla})$, enones $(\boldsymbol{\nabla})$, and dicarbonyls $(\bullet)$. 
The $\mathcal{T}_{1}$ diagnostics in Table $\mathrm{S} 4$ for the TDCAM-B3LYP $S_{1}$ minimum energy structures are all below the 0.02 threshold considered acceptable for closed-shell singlet calculations. ${ }^{78}$ For $S_{1}$ NTII TS geometries several $\mathcal{T}_{1}$ diagnostic values were at or above the 0.02 threshold: pentanal (0.055), hex-3-enal (0.041), 4-methylpentan-2one (0.027), hex-4-en-3-one (0.022), and but-2enal (0.020). In all of these species the $S_{1}-S_{0}$ energy gaps are $<100 \mathrm{~kJ} / \mathrm{mol}$, as determined by 10 -root TD-CAM-B3LYP single point energy calculations. The $S_{1}-S_{0}$ separations for but-2-enal, hex-3-enal, and hex-4-en-3-one are $<30 \mathrm{~kJ} / \mathrm{mol}$. These energy gaps are lower than observed for species with acceptable $\mathcal{T}_{1}$ diagnostics, with the exception of pentan-2-one which has a low $(65 \mathrm{~kJ} / \mathrm{mol})$ energy gap without a $\mathcal{T}_{1}$ diagnostic above 0.02 for the $S_{1}$ NTII TS.

None of the $S_{1}$ NTII intrinsic reaction barriers for these species with poor $\mathcal{T}_{1}$ values were identified, from Figure 2, as being potentially problematic. Indeed, there is also no indication, on the $\mathcal{T}_{1}$ diagnostics presented in Table $\mathrm{S} 4$, that the $S_{1}$ calculations for butanal, 3-methylpentanal, hexan-2-one, hexan-3-one, hex-1-en-3-one, and 2-oxobutanal are problematic, despite their inconsistent EOM-CCSD energies in Figure 2. It appears a poor $\mathcal{T}_{1}$ diagnostic is not necessarily an indication that the $S_{1}$ NTII energy calculations by TD-DFT or EOM-CCSD may be unreliable. The converse also does not hold, an acceptable $\mathcal{T}_{1}$ diagnostic cannot be taken as assurance that the $S_{1}$ energy is reliable. All linear response $S_{1}$ calculations for the NTII reaction should therefore be treated with caution.

\section{$S_{1} / S_{0}$ conical intersections}

MECI searches were performed on species with large $\mathcal{T}_{1}$ diagnostic values, as well as for butanal, hexanal, and 2-oxobutanal. This ensured that all classes of carbonyl were considered, to determine whether an $S_{1} / S_{0}$ MECI in the vicinity of the $S_{1}$ NTII reaction coordinate is a feature common across all carbonyl classes. While the energetics of CASSCF will be poor due to lack of dynamic electron correlation, the geometries should be reliable as CASSCF bond lengths are found to differ by $\sim 0.01-0.02 \AA$ com- pared to CASPT2 geometries for small organic molecules, including saturated and unsaturated carbonyls. ${ }^{80}$

The optimised $S_{1} / S_{0}$ MECIs for 4methylpentan-2-one and 2-oxobutanal, are overlaid on their corresponding $S_{1}$ NTII TS structures in Figure 3. These two examples are representative of all species, and similar figures for all other MECIs are available in Figure S3 of the supporting information. The striking similarity of the $S_{1} / S_{0}$ MECIs for all carbonyls considered suggests similar $S_{1} / S_{0}$ MECIs will be present for all carbonyls that bear a $\gamma$-hydrogen. This mechanism is therefore likely to be important in all tropospheric NTII photolysis reactions.

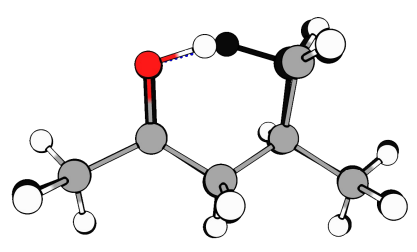

(a) 4-Methylpentan-2-one

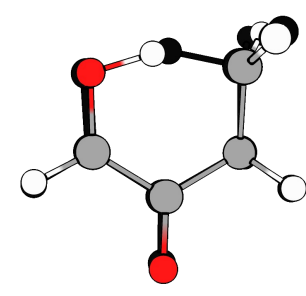

(b) 2-Oxobutanal
Figure 3: Structures of the $S_{1} / S_{0}$ MECIs (coloured) overlaid on the $S_{1}$ NTII TS structures (black silhouette). MECIs are calculated at the $\operatorname{CAS}(10,8) / 6-31 \mathrm{G}(\mathrm{d})$ level of theory, while $S_{1}$ NTII TS structures are from CAMB3LYP/def2-TZVP calculations.

For all carbonyls considered, the $S_{1} / S_{0}$ MECI has a very similar structure to the $S_{1}$ NTII TS, and is on the 1,4-biradical product side of the TS. This is the reaction coordinate for $S_{1}$ NTII reactions. These calculations therefore support the generic $S_{1}$ NTII mechanism shown in Figure 1, where crossing to $S_{0}$ occurs before the biradical intermediate is formed, implying short $S_{1}$ lifetimes.

Despite the presence of $S_{1} / S_{0}$ MECIs in the vicinity of $S_{1} \mathrm{TS}$, and the observation of problematic $\mathcal{T}_{1}$ diagnostics and small $S_{1}-S_{0}$ energy gaps, no clear approach is apparent for determining whether the calculated $S_{1}$ NTII photolysis thresholds are reliable. Perhaps the best available indication is the similarity of calculated TDB2GP-PLYP $S_{1}$ energies to the trends observed for the $T_{1}$ intrinsic reaction barriers, as was shown in Figure 2. In terms of literature precedent, there are suggestions that time-dependent 
double-hybrid DFT methods are more reliable in the vicinity of CIs for acetophenone ${ }^{81}$ due to the treatment of double excitations, ${ }^{58,82}$ and this is broadly supported in Figure 2. Therefore TD-B2GP-PLYP $S_{1}$ NTII intrinsic reaction barriers were reported in Table 1, and are used below in the discussion of the NTII reaction on different electronic states.

\section{Discussion}

\section{The $S_{1}$ NTII reaction}

The $S_{1}$ NTII photolysis threshold energies from Table 1 are shown in Figure 4, alongside a dashed blue line denoting the $313 \mathrm{~nm}$ photolysis energy used in NTII triplet quenching studies of butanal, pentan-2-one, and hexan-2-one. ${ }^{6,7} \mathrm{~A}$ solid purple line at $400 \mathrm{~kJ} / \mathrm{mol}$ is used to indicate an approximate maximum photon energy in the troposphere in the figures below. As discussed previously, the $S_{1}$ photolysis thresholds in Figure 4 are consistent with experimental singlet/triplet ratios.

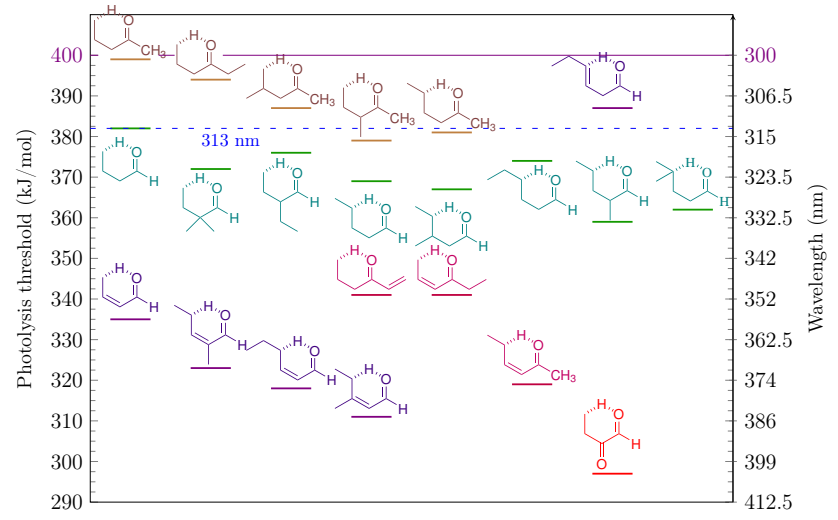

Figure 4: Photolysis thresholds for the $S_{1}$ NTII reaction, calculated at the TD-B2GP-PLYP//TD-CAMB3LYP $+\mathrm{CC} / \mathrm{DFT}^{\prime \prime}$ level of theory (see text).

The calculated $S_{1}$ NTII thresholds of ketones are $\sim 10 \mathrm{~kJ} / \mathrm{mol}$ higher than the equivalent aldehyde species, reflective of higher $S_{0} \rightarrow S_{1} 0_{-0}$ excitation energies since the intrinsic barriers are similar. The $\alpha, \beta$-unsaturated carbonyls are predicted to have the lowest $S_{1} 1,5-\mathrm{H}$-shift photolysis thresholds, due to their low $0-0$ excitation energies. The enals are calculated to have slightly higher $S_{1}$ NTII thresholds than the enones, again due to differences in $S_{0} \rightarrow S_{1}$ $0-0$ excitation energies, since the $S_{1}$ intrinsic reaction barriers calculated for enals are low, or even barrierless. Because hex-3-enal is $\beta, \gamma$ unsaturated there is no conjugation with the $\mathrm{C}=\mathrm{O}$ chromophore in this molecule, and hence its excitation energies and photolysis thresholds resemble those of the saturated carbonyls. 2-Oxobutanal is predicted to have a low (294 $\mathrm{kJ} / \mathrm{mol}) S_{1}$ NTII photolysis threshold due to low excitation energies in $\alpha$-dicarbonyls.

From Figure 4, the $S_{1}$ NTII photolysis thresholds of the saturated ketones are within $\sim 20$ $\mathrm{kJ} / \mathrm{mol}$ of the maximum photon energy available in the troposphere $(\sim 400 \mathrm{~kJ} / \mathrm{mol})$. Since the intensity of solar radiation increases with wavelength, this means that $S_{1}$ NTII reactions of saturated ketones in the troposphere are likely to be slow, with small QYs. In saturated carbonyls, ISC to $T_{1}$ and reaction on $T_{1}$ will be competitive, if not the dominant photolysis pathway. Exceptions occur for saturated carbonyls whose $S_{1}$ NTII photolysis thresholds are lowered by substitution. The SARs dictating the lowering of photolysis thresholds with substitution patterns will be elucidated after discussion of NTII photolysis thresholds calculated on each electronic state.

\section{The $T_{1}$ NTII reaction}

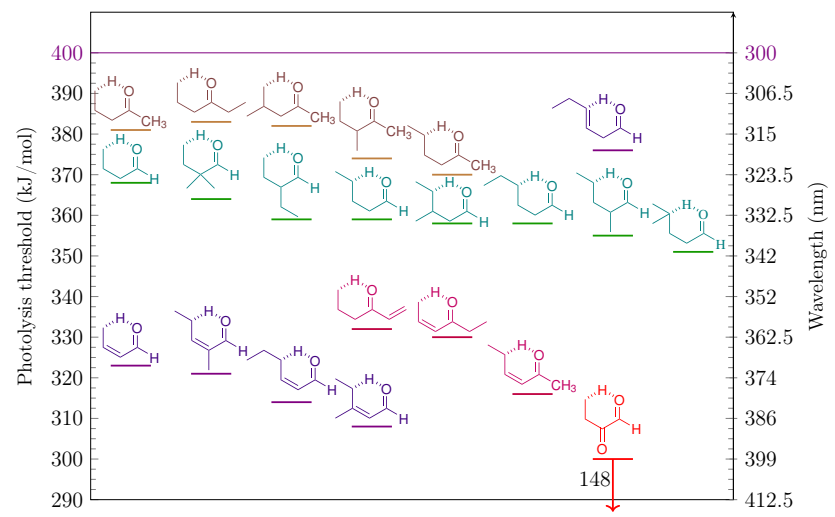

Figure 5: Photolysis thresholds of the $T_{1}$ NTII reaction, calculated at the UB2GP-PLYP/def2-TZVP level of theory.

The calculated $T_{1}$ NTII photolysis thresholds are shown in Figure 5. Comparison of Figures 4 and 5 shows the same general relationships 
within and between carbonyl classes for both $S_{1}$ and $T_{1}$ NTII thresholds. However, all $T_{1}$ NTII thresholds are predicted to be lower than 380 $\mathrm{kJ} / \mathrm{mol}(315 \mathrm{~nm})$, meaning that the $T_{1}$ NTII reaction pathways should be accessible in the previous $313 \mathrm{~nm}$ or $315 \mathrm{~nm}$ photolysis experiments on butanal, pentanal, pentan-2-one, and hexan-2-one. ${ }^{6,7,32}$

In principle, all $T_{1}$ NTII reactions are accessible under tropospheric conditions for all carbonyls considered here. Moreover, even at the lowest actinic energies at which saturated carbonyls absorb (for aldehydes this is $\sim 360$ $\mathrm{kJ} / \mathrm{mol}),{ }^{83}$ the $T_{1}$ NTII photolysis channel is open for many carbonyls. This will lead to relatively large $T_{1}$ NTII QYs within the actinic range for most large carbonyls. This will also make experimental measurement of $T_{1}$ NTII photolysis thresholds in aldehydes difficult, as the UV absorption energies that can be accessed in an experiment will be above the $T_{1}$ NTII threshold. Similarly, the predicted $T_{1}$ NTII photolysis threshold of 2-oxobutanal, at $148 \mathrm{~kJ} / \mathrm{mol}$, will prevent a precise $T_{1}$ NTII photolysis threshold measurement.

The ketones are predicted to have $T_{1}$ NTII photolysis thresholds $\sim 10 \mathrm{~kJ} / \mathrm{mol}$ higher than equivalent aldehydes, and so their $T_{1}$ thresholds are above $360 \mathrm{~kJ} / \mathrm{mol}(330 \mathrm{~nm})$. Therefore, photolysis experiments on ketones with lower energy wavelengths, $\sim 330 \mathrm{~nm}$ or longer, should show the photolysis behaviour of these species when the $T_{1}$ NTII reaction is inaccessible.

The enals and enones are predicted to have low $T_{1}$ NTII photolysis thresholds, at or below 330 $\mathrm{kJ} / \mathrm{mol}$. The $\alpha, \beta$-unsaturated carbonyls have broader absorption cross-sections than saturated species - the longest wavelength at which typical enals and enones absorb is $\sim 400 \mathrm{~nm}(\sim 300$ $\mathrm{kJ} / \mathrm{mol}) .{ }^{83}$ Although the $T_{1}$ NTII photolysis thresholds are energetically accessible in $\alpha, \beta$ unsaturated carbonyls, as will be discussed below, the second step of the NTII reaction is not possible in these species.

\section{The NTII reaction in $\alpha, \beta$-unsaturated carbonyls}

Of the enals, the photolysis of but-2-enal (crotonaldehyde) is the most experimentally studied. ${ }^{84-89}$ None of these studies, however, have detected the enol and alkene photoproducts which would be expected from the NTII reaction, despite the low predicted $S_{1}$ and $T_{1}$ NTII photolysis thresholds shown in Figures 4 and 5 . This can be rationalised by the inability of $\alpha, \beta$ unsaturated species to cleave their $\alpha, \beta$-bond after formation of the 1,4-biradical. The photolysis threshold energies shown in Figures 4 and 5 are for the initial 1,5-H-shift, but, as shown in Scheme 4, the $\alpha, \beta$-double-bond prevents cleavage from occurring following electron migration to stabilise the $\gamma-\mathrm{C}^{\bullet}$ and ${ }^{\circ} \mathrm{C}-\mathrm{O}$ radical centres.

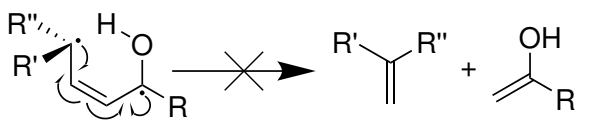

Scheme 4: $\alpha, \beta$-unsaturation prevents Norrish Type II bond cleavage to an alkene and an enol.

Instead, photoisomerisation dominates the photolysis of but-2-enal. ${ }^{85,88,89}$ Based on the inability of $\alpha, \beta$-unsaturated species to undergo NTII reaction, and the high $T_{1}$ NTI $a$ photolysis thresholds calculated for unsaturated species in earlier work, ${ }^{49}$ ground state reactions and isomerisations are expected to dominate the photolysis of all $\alpha, \beta$-unsaturated species.

\section{The $S_{0}$ concerted NTII reaction}

There have been proposals of an alternative $S_{0}$ concerted NTII reaction where the 6 -membered cyclic TS leads directly to alkene and enol formation in one step, ${ }^{16}$ as shown in Figure 6 a. Analogous reaction mechanisms are well documented in organic chemistry in the class of 'retro-ene' pericyclic sigmatropic reactions ${ }^{90,91}$ (Figure 6c), and the McLafferty rearrangement in mass spectrometry ${ }^{92}$ (Figure 6b).

Complete retention of stereochemistry should be seen in concerted $S_{0}$ NTII reaction products, by analogy to suprafacial migration in $[1,5]$ sigmatropic rearrangements. ${ }^{90}$ However, stereochemistry alone is not sufficient to disambiguate 
${ }^{\mathrm{R}}$

(a) Concerted

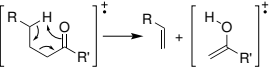

(b) McLafferty

(c) Retro-ene

Figure 6: The reaction schemes for the proposed concerted $S_{0}$ NTII reaction (a), alongside the McLafferty rearrangement (b), and the retro-ene reaction (c).

between $S_{1}$ or $S_{0}$ NTII reactions, since the $S_{1} / S_{0}$ conical intersection promotes cleavage following an $S_{1}$ 1,5-H-shift before stereochemical scrambling can occur. Evidence for $S_{0}$ NTII reactions therefore relies on energetic arguments, reinforcing the importance of accurate prediction of $S_{1}$, $T_{1}$, and $S_{0}$ NTII photolysis threshold energies.

We calculate the reaction thresholds for the $S_{0}$ NTII reaction, which is a concerted reaction where the intramolecular hydrogen transferred is accompanied by $\alpha, \beta$-bond breaking in the same step. These $S_{0}$ reaction thresholds are shown in Figure 7, and have been labelled as 'photolysis thresholds' to emphasise that, although these energies are $S_{0}$ intrinsic reaction barriers, $S_{0}$ NTII reactions under tropospheric conditions would occur by absorption of actinic photons. Concerted $S_{0}$ NTII TSs could not be located for most enals and enones: the double-bonds at the $\alpha, \beta$-position in these species prevent $\alpha, \beta$-bond cleavage from accompanying the 1,5-H-shift.

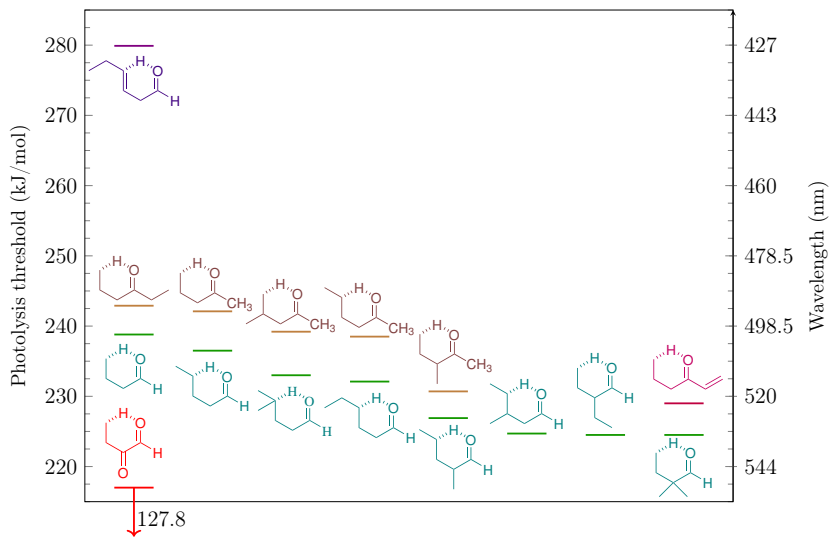

Figure 7: Photolysis thresholds for the concerted $S_{0}$ NTII reaction, calculated at the B2GP-PLYP/def2TZVP level of theory.

The $S_{0}$ NTII photolysis thresholds shown in Figure 7 are all well below the longest wavelength range where saturated carbonyls absorb $(\sim 330 \mathrm{~nm} / 362.5 \mathrm{~kJ} / \mathrm{mol}){ }^{83}$ If formation of NTII photoproducts in pentan-2-one in recent
$330 \mathrm{~nm}$ FT-IR experiments by Kharazmi is evidence for the existence of a $S_{0}$ NTII pathway, ${ }^{32}$ then the thresholds shown in Figure 7 indicate that energetically accessible $S_{0}$ NTII reaction pathways should be available for all saturated carbonyl species. Hex-3-enal is predicted to have the highest concerted $S_{0}$ NTII photolysis threshold, since the $\gamma-\mathrm{H}$ transferred is adjacent to the $\beta, \gamma$-double-bond and so the energy of the closed-shell TS to transfer the $\gamma-\mathrm{H}$ is raised. Apart from hex-3-enal, the concerted $S_{0}$ NTII threshold energies of all other carbonyls are similar, within an $\sim 225-245 \mathrm{~kJ} / \mathrm{mol}$ range.

A master equation scheme for butanal photolysis proposed by Shaw $^{26}$ included the concerted $S_{0}$ NTII pathway, but this pathway was found to be inconsequential at 290-330 nm photolysis wavelengths. So, while the $S_{0}$ NTII pathway should be energetically accessible in butanal, it is predicted to be kinetically unimportant where $S_{1}$ and $T_{1}$ channels are open. In contrast, recent $330 \mathrm{~nm}$ pentan-2-one photolysis data by Kharazmi indicates that an $S_{0}$ NTII reaction is occurring in pentan-2-one. ${ }^{32}$ As seen in Figure $7, S_{0}$ NTII photolysis threshold energies do not explain the different photochemical behaviour of butanal and pentan-2-one, since the thresholds for both molecules are significantly lower than the photolysis energies used.

Alternatively, the predicted $T_{1}$ NTII threshold for pentan-2-one could be wrong, leading to miss-assignment of the electronic state involved, though a $15 \mathrm{~kJ} / \mathrm{mol}$ overestimate by the UB2GPPLYP/def2-TZVP calculations seems outside of the accuracy demonstrated when benchmarking to experimental $T_{1}$ thresholds, ${ }^{49}$ and is in good agreement with other estimates of the $T_{1}$ thresholds. ${ }^{32}$ If another photolysis pathway apart from the NTII reaction could form ethene, it could explain the QYs at $330 \mathrm{~nm}$, however our own calculations exploring other possible ground state reactions do not present a viable alternative pathway.

The master equation calculations of Shaw on butanal indicate that, from 310-330 nm, NTIa reactions on either $T_{1}$ or $S_{0}$ dominate. It is possible that the $S_{0}$ NTII reaction is observed for pentan-2-one and not butanal because the $T_{1}$ NTI $a$ and NTII photolysis thresholds are 
higher for pentan-2-one than for butanal, ${ }^{49}$ and so reaction on $T_{1}$ is closed at longer wavelengths for pentan-2-one, allowing significant ISC to $S_{0}$ and an appreciable amount of $S_{0}$ NTII reactions.

\section{SARs for Excited State NTII Reactions}

The SARs elucidated in the following subsections are derived from $T_{1}$ NTII photolysis energies. Since both the $S_{1}$ and $T_{1}$ states arise from $n \rightarrow \pi^{*}$ excitations, and the $S_{1}$ and $T_{1}$ carbonyl biradicals have similar structures and electron distributions, these SARs should also apply to $S_{1}$ NTII photolysis. They are not, however, applicable to concerted $S_{0}$ NTII reactions. Since the $S_{0}$ NTII photolysis thresholds are predicted to be below actinic energies, a simple SAR for $S_{0}$ NTII pathways is to assume that they are accessible in the troposphere for all but $\alpha, \beta$-unsaturated carbonyl species.

\section{Substitution at the $\gamma$-position}

Substitution at the $\gamma$-position is the clearest SAR for excited state NTII processes, leading to a significant decrease in excited state photolysis thresholds. For instance, upon moving from butanal with a primary $\gamma$-carbon, to pentanal with a secondary $\gamma$-carbon, the $T_{1}$ NTII threshold drops by $\sim 10 \mathrm{~kJ} / \mathrm{mol}$. This $\gamma$-substitution effect in carbonyl NTII photolysis is consistent with triplet quenching experiments: at $313 \mathrm{~nm}$ pentan-2-one NTII photolysis is a triplet process, whereas it is a singlet process in hexan-2one ${ }^{6}$ since the extra $\gamma$-substituent lowers the $S_{1}$ NTII threshold to become energetically accessible. This stabilisation is due to hyperconjugation of the incipient 1,4-biradical at the position of $\gamma-\mathrm{H}$ abstraction, as illustrated in Figure 8 . Similar to the relatively late transition states in the NTI $a$ and NTI $b$ reactions, ${ }^{49}$ NTII photolysis thresholds are also stabilised through hyperconjugation.

Further chain extension, e.g. moving from pentanal to hexanal, does not further decrease the $T_{1}$ NTII threshold. However additional substitution, such as the tertiary $\gamma$-carbon centre in
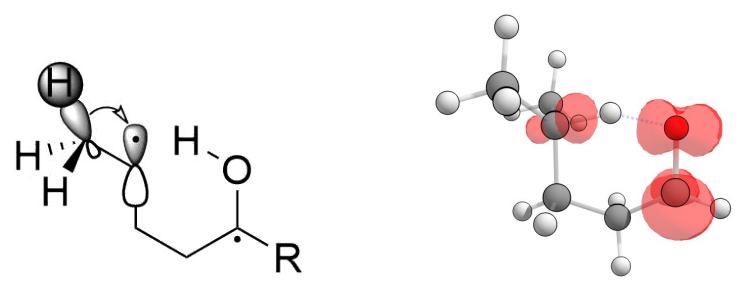

(a) Hyperconjugation at (b) 4-methylpentanal $T_{1}$ the $\gamma$-position. NTII TS.

Figure 8: Substitution at the $\gamma$-position directly stabilises the incipient 1,4-biradical in the NTII reaction. This is illustrated in the Lewis structure in (a), and shown in (b) through the unpaired electron density of the $T_{1}$ NTII TS calculated for 4-methylpentanal.

4-methylpentanal, drops the threshold by $\sim 20$ $\mathrm{kJ} / \mathrm{mol}$ compared to the primary $\gamma$-carbon in butanal.

A simple SAR for excited state NTII reactions is that the photolysis threshold will decrease by $\sim 10 \mathrm{~kJ} / \mathrm{mol}$ per extra degree of substitution at the $\gamma$-position. This effect is seen across the other carbonyl classes in Figure 5, including the ketones ( $c f$. pentan-2-one and hexan-2-one), the enals ( $c f$. but-2-enal and hex-2-enal), and the enones ( $c f$. hex-4-en-3-one and hex-3-en-2-one).

\section{Substitution at carbonyl, $\alpha$, and $\beta$ positions}

The incipient radical position in the ${ }^{\circ} \mathrm{C}-\mathrm{O}$ moiety of the forming biradical could also be stabilised by hyperconjugation. Alkyl substitution at the carbonyl-carbon should provide adjacent $\mathrm{C}-\mathrm{H} \sigma$-bonds for hyperconjugation. However, the calculated NTII intrinsic reaction barriers for ketones in Table 1 are typically raised compared to equivalent aldehydes, rather than lowered. This indicates that any potential hyperconjugative stabilisation gained by the ketone alkyl group is offset by destabilisation of the excited $\mathrm{C}=\mathrm{O}$ chromophore by the alkyl auxochrome.

For substitution at the $\alpha$-position, $T_{1}$ thresholds in Figure 5 do not indicate a clear effect. The $T_{1}$ NTII photolysis threshold of 2-methylpentanal is predicted to be lowered by $4 \mathrm{~kJ} / \mathrm{mol}$ compared to $\alpha$-unsubstituted pentanal. Similarly, the $T_{1}$ threshold for $3-$ methylpentan-2-one is lowered $7-9 \mathrm{~kJ} / \mathrm{mol}$ compared to $\alpha$-unsubstituted ketones. However, 
the lack of NTII threshold energy lowering in the $T_{1}$ intrinsic NTII barrier of doubly- $\alpha$ substituted 3,3-dimethylbutanal (though threshold lowering is seen on $S_{1}$ ) indicates that any $\alpha$-substitution effect is not as straightforward as the $\gamma$-substitution effect

Substitution at the $\alpha$-position should deliver no hyperconjugative stabilisation of the $\mathrm{C}^{\bullet}$ center if it was strictly localised as in a simple Lewis structure (Figure 9a). However the $\alpha$-carbon does show a small amount of spin density delocalisation at a low isosurface value of 0.005 (Figure $9 \mathrm{~b}$ ), which could confer some of this $T_{1}$ NTII barrier lowering predicted for $\alpha$-substituted species.

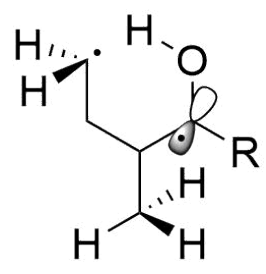

(a) Lewis structure with lo- (b) 2-methylpentanal $T_{1}$ calised radical centres. NTII TS.

Figure 9: Substitution at the $\alpha$-position may indirectly stabilise unpaired ${ }^{\circ} \mathrm{C}-\mathrm{O}$ electron density in the incipient 1,4-biradical in the NTII reaction. This is not shown by the Lewis structure of 2-methylpentanal in (a), but a small indication can be seen in the unpaired spin density of 2-methylpentanal (b) at an isosurface value of 0.005 .

Energy differences of $\sim 4 \mathrm{~kJ} / \mathrm{mol}$ are however at the limit of chemical accuracy, and so using such differences to infer SARs is tentative. Additionally, given the limited number of $\beta$ substituted species shown in Figure 5, there is insufficient data to draw a SAR based on substitution at the $\beta$-position. It would be expected that $\beta$-substitution affects NTII thresholds similarly to $\alpha$-substitution, since both place an alkyl chain two carbons away from a biradical centre.

These SARs hold for alkyl substituents, and not substituents which are strongly electron withdrawing or donating. ${ }^{16}$ While substituents at the $\alpha$ or $\beta$ position do not strongly alter energetic thresholds, they do affect the preferred conformation of the resulting biradical and hence influence the ratio of cyclisation vs. cleavage products of the NTII reaction. ${ }^{16,93,94}$ For aryl carbonyls, substitution at $\alpha$ and $\beta$ positions of aromatic rings can alter NTII reactivity, as was observed in photolysis studies of substituted valerophenones. ${ }^{93}$ Since aryl carbonyls are not common in the troposphere, they are excluded from this study and SARs pertaining to aromatic carbonyls are not determined here.

\section{$\alpha, \beta$-Unsaturation and allylic radi- cal resonance.}

$\alpha, \beta$-Unsaturated species display lower $S_{1}$ and $T_{1}$ intrinsic reaction barriers in Table 1 than the saturated carbonyls. This can be rationalised by considering allylic radical resonance stabilisation. ${ }^{95}$ From the perspective of Lewis structures, unsaturation near a radical site allows multiple resonance contributors, as shown in Figure 10a. This allylic radical stabilisation manifests as delocalisation of the unpaired spin density, shown for the example of the hex-4-en-3-one $T_{1}$ NTII TS in Figure 10b. In Figure 10b, the spin density has delocalised to the $\beta$-position; this is not seen in saturated carbonyls.

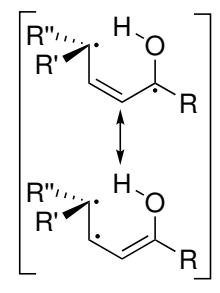

(a) Allylic radical resonance stabilisation.

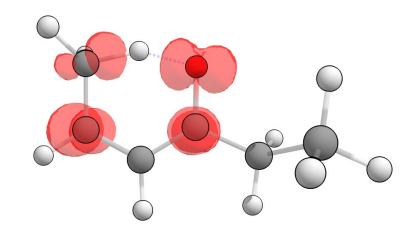

(b) Hex-4-en-3-one $T_{1}$ NTII TS.
Figure 10: Unsaturated near incipient radical sites formed in the NTII reaction will lower reaction barriers through allylic resonance stabilisation. Lewis structure resonance contributors are illustrated in (a), whereas the spin density of the hex-4-en-3-one $T_{1}$ NTII TS shown in (b) demonstrates that the unpaired electron density delocalises over to the $\beta$-position.

The lowering of excited state NTII intrinsic reaction barriers by allylic radical resonance stabilisation in $\alpha, \beta$-unsaturated carbonyls is significant, approximately $15-20 \mathrm{~kJ} / \mathrm{mol}$ lower compared to corresponding saturated carbonyls. For instance, the intrinsic $T_{1}$ NTII barrier for but-2-enal is $18 \mathrm{~kJ} / \mathrm{mol}$ lower than for butanal, in hex-3-en-2-one the barrier is $14 \mathrm{~kJ} / \mathrm{mol}$ lower compared to hexan-2-one, and in hex-2-enal the barrier is $23 \mathrm{~kJ} / \mathrm{mol}$ lower than in hexanal. 
A simple SAR to account for this radical allylic resonance is to posit that $\alpha, \beta$-unsaturation leads to $15-20 \mathrm{~kJ} / \mathrm{mol}$ lower intrinsic excited state NTII barriers, compared to the corresponding saturated carbonyl. Combined with the SAR of an approximately $30-50 \mathrm{~kJ} / \mathrm{mol} 0-0$ excitation energy decrease in $\alpha, \beta$-unsaturated carbonyls, this leads to excited state NTII 1,5-Hshift photolysis thresholds in $\alpha, \beta$-unsaturated carbonyls $\sim 50 \mathrm{~kJ} / \mathrm{mol}$ lower than the equivalent saturated species.

\section{Barrierless, or small barrier, ex- cited state NTII reactions}

The intrinsic reaction barriers for the excited state NTII 1,5-H-shift photolysis thresholds in Table 1 are mostly under $30 \mathrm{~kJ} / \mathrm{mol}$ on $S_{1}$ and under $50 \mathrm{~kJ} / \mathrm{mol}$ on $T_{1}$. If hex-3-enal is removed, because its $\beta, \gamma$-unsaturation raises abstraction barriers, the least substituted saturated carbonyls, such as butanal, 3,3-dimethylbutanal, and hexan-3-one, have the highest intrinsic reaction barriers. As above, $\alpha, \beta$-unsaturation is predicted to lower NTII intrinsic reaction barriers by $\sim 15-20 \mathrm{~kJ} / \mathrm{mol}$, and barriers should decrease by $\sim 10 \mathrm{~kJ} / \mathrm{mol}$ for each extra degree of $\gamma$-substitution. Since the $S_{1}$ NTII intrinsic barriers for butanal and pentan-2-one are predicted to be $\sim 30 \mathrm{~kJ} / \mathrm{mol}$, lowering of the photolysis energy due to the SARs above leads to predictions of small barriers, or even barrierless processes, for carbonyl species that are both $\alpha, \beta$-unsaturated and substituted at the $\gamma$-position. For example, the butanal $S_{1}$ NTII intrinsic reaction barrier is predicted to be 29 $\mathrm{kJ} / \mathrm{mol}$, but the but-2-enal intrinsic reaction barrier is significantly smaller, $\sim 5 \mathrm{~kJ} / \mathrm{mol}$, due to the $\alpha, \beta$-unsaturation. When combined with extra $\gamma$-substitution, the $S_{1}$ NTII intrinsic reaction barriers in enals become negative, i.e. are submerged barriers. ${ }^{96}$ This includes: hex-2-enal, 2-methylpent-2-enal, and 3-methylpent-2-enal in Table 1. This means that as long as photoexcitation to the $S_{1}$ minimum is possible, the $S_{1}$ NTII 1,5-H-shift should always be energetically accessible in these species.

The XMCQDPT2//CASSCF multiconfigurational calculations by Kletskii et al. predict a negative $S_{1}$ intrinsic reaction barrier for but-2enal, while in Table 1 this barrier is predicted to be slightly positive. However, in larger enals, substitution at the $\gamma$-position should lower the intrinsic reaction barrier further and thus be predicted to be negative. Therefore, these calculations present a model that $\gamma$-substituted enals may have barrierless $S_{1}$ NTII 1,5-H-shifts, with small barriers for enones.

It would be informative to study the entire $S_{1}$ NTII reaction pathway of these species with accurate multiconfigurational methods, which has recently been made possible with the development of analytic CASPT2 nuclear gradients. ${ }^{97}$ However, as noted before, these $\alpha, \beta$ unsaturated species are unlikely to undergo NTII cleavage, but instead will react through ground state isomerisation or dissociation pathways.

\section{Conclusions}

The calculations in this paper predict the energetics of $S_{1}, T_{1}$, and $S_{0}$ NTII reaction pathways in 22 carbonyl species and confirm the general schematic introduced in Figure 1: when $S_{1}$ photolysis thresholds are energetically accessible due to $\gamma$-substitution then formation of a $S_{1}$ biradical is rapid, the biradical species then crosses to $S_{0}$ via an $S_{1} / S_{0}$ conical intersection and cleaves to form an $S_{0}$ alkene and an enol. When the $S_{1}$ NTII threshold is inaccessible, then ISC to $T_{1}$ and formation of a long-lived triplet biradical occurs. The triplet biradical can then cleave or cyclise. While $\alpha, \beta$-unsaturated carbonyls are all predicted to have very low NTII thresholds for the 1,5-H-shift reaction because of allylic radical stabilisation, these species cannot cleave the $\alpha, \beta$-bond. Instead, it is likely they cross to $S_{0}$ as internally 'hot' molecules. On $S_{0}$, the major unimolecular reaction pathways of these species are expected to be ground state isomerisation and dissociation reactions. Concerted $S_{0}$ pathways to cleavage to an alkene and an enol are calculated for most saturated species, and could explain the observation of ethene production from pentan-2-one at $330 \mathrm{~nm} .{ }^{32}$ This concerted $S_{0}$ NTII pathway is also predicted 
to be energetically accessible for the other carbonyls considered here. The reason this pathway has not been experimentally observed in other carbonyl species may be because reaction on $S_{1}$ and $T_{1}$ dominates at the experimental photolysis energies considered.

The NTII reaction is important in atmospheric models since 5-6 carbon species are common biogenic VOCs, ${ }^{40,98}$ which are oxidised in the atmosphere into carbonyls that can undergo the NTII reaction. However, the treatment of the NTII reaction in atmospheric chemistry models is inconsistent. The NYC reaction is not included in the photolysis schemes of major atmospheric chemistry models. NYC products are observed as a minor channel of $T_{1}$ NTII photolysis of gas-phase carbonyls. More pressingly, the NTII QY estimated for carbonyls is different between the two major atmospheric models (the $\mathrm{MCM}^{31}$ and GEOS-Chem ${ }^{99}$ ), and both models are inconsistent with the experimental data and the photolysis thresholds calculated here.

For example, the MCM uses a NTII rate measured for butanal as the 'surrogate' rate for the wider array of carbonyls which undergo NTII reaction. ${ }^{31,100}$ GEOS-Chem is less detailed: the NTII reaction is excluded entirely from the generic photolysis scheme of aldehydes containing more than three carbon atoms. ${ }^{99}$

The results of this paper show that using a generic NTII rate constant from butanal is inappropriate. Butanal should set a lower limit for NTII reaction QYs in aldehydes, as the SARs determined here predict that more substituted carbonyls should have lower NTII photolysis thresholds. Indeed, in 290-330 nm photolysis experiments (observing $\mathrm{HCO}^{\circ}$ yields) the NTII QY was inferred to range from $0.52-0.56$ for butanal, but was $0.68-0.91$ for pentanal. ${ }^{101}$

The calculations in this paper characterised the NTII photolysis thresholds on all relevant electronic states, and located $S_{1} / S_{0}$ MECIs, for carbonyl species representative of each carbonyl class. There was no clear diagnostic for the failure of linear response methods near the vicinity of a MECI, however $S_{1}$ energies computed with time-dependent double-hybrid methods were consistent with the substituent effects on $T_{1}$ energies. These calculations provide compre- hensive insight into the energetics of the NTII reaction. However, rationalising experimental data on the prevalence of cyclisation, cleavage, or H-backtransfer from the 1,4-biradical requires dynamic modelling. $8,12,35,94,102$ More theoretical studies of the NTII biradical ${ }^{42}$ are required, and $a b$ initio dynamics has only been performed for the NTII reaction in pentanal. ${ }^{43,44}$ Furthermore, in order to assess the competition between NTII and other photolysis reactions on $S_{1}, T_{1}$, and $S_{0}$ kinetic modelling is required. The reaction energies, TS structures, and $S_{1} / S_{0}$ MECIs reported in this paper can be used as the basis for master equation calculations for NTII reactions across many carbonyl species.

Acknowledgement This work was supported by the Australian Research Council (grant DP160101792). It was also supported by grants of computer time under the Merit Allocation Scheme on the NCI National Facility at the Australian National University, as well as computer time on the computational cluster Katana supported by the Faculty of Science, UNSW Australia, and the computational cluster Artemis supported by the Sydney Informatics Hub at the University of Sydney. KNR recognises an Australian Government Research Training Program (RTP) scholarship.

\section{Supporting Information Avail- able}

The Supporting Information contains: comparison between linear response $S_{1}$ NTII intrinsic reaction barriers and $S_{1}$ NTII intrinsic reaction barriers published in the literature; details of the active spaces used in CASSCF calculations; $S_{0} \rightarrow S_{1}$ excitation energies calculated on $S_{1}$ minima and transition states using a range of linear response methods; visual overlays of $S_{1} / S_{0}$ minimum energy conical intersections and $S_{1}$ NTII transition states; diagnostic $\mathcal{T}_{1}$ values and $S_{2}-S_{1}$ excited state energy separations. (PDF)

Cartesian co-ordinates for all optimised structures (ZIP) 


\section{References}

(1) Wagner, P. J.; Zepp, R. G. Trapping by mercaptans of biradical intermediates in type II photoelemination. J. Am. Chem. Soc. 1972, 94, 287, DOI: 10.1021/ja00756a057.

(2) O’Neal, H. E.; Miller, R. G.; Gunderson, E. Quenching by Hydrogen Bromide of the Norrish Type II Process in the Photolysis of 2-Pentanone. Chemical Trapping of a Triplet 1,4 Biradical in the Gas Phase. $J$. Am. Chem. Soc. 1974, 96, 3351-3358, DOI: 10.1021/ja00818a003.

(3) Wagner, P. J.; Klán, P. CRC Handbook of Organic Photochemistry and Photobiology, 2nd Edition; 2004; Chapter 52, pp 1-22.

(4) Tadić, J. M.; Juranić, I. O.; Moortgat, G. K. Photooxidation of n-heptanal in air: Norrish type I and II processes and quantum yield total pressure dependency. J. Chem. Soc. Perkin Trans. 2 2002, 2, 135-140, DOI: 10.1039/b106476m.

(5) Tadić, J. M.; Moortgat, G. K.; Bera, P. P.; Loewenstein, M.; Yates, E. L.; Lee, T. J. Photochemistry and photophysics of n-butanal, 3-methylbutanal, and 3,3-dimethylbutanal: Experimental and theoretical study. J. Phys. Chem. A 2012, 116, 5830-5839, DOI: $10.1021 /$ jp208665v.

(6) Michael, J. L.; Noyes, W. A. The Photochemistry of Mixtures of 2-Pentanone and 2-Hexanone with Biacetyl. J. Am. Chem. Soc. 1963, 85, 1027-1032, DOI: 10.1021/ja00891a001.

(7) Tölgyesi, M.; Nacsa, A.; Bérces, T. Quenching of the photochemical decomposition of n-butyraldehyde in the vapour phase and in isooctane. J. Photochem. 1979, 11, 333-345, DOI: 10.1016/0047-2670(79)85022-4.

(8) Stephenson, L. M.; Cavigli, P. R.; Parlett, J. L. Chemistry of 1,4-diradicals. I. Intermediates in the Norrish type II photocleavage. J. Am. Chem. Soc. 1971, 93, 1984-1988, DOI: $10.1021 /$ ja00737a023.

(9) Nau, W. M.; Greiner, G.; Wall, J.; Rau, H.; Olivucci, M.; Robb, M. A. The Mechanism for Hydrogen Abstraction by $n, \pi^{*}$ Excited
Singlet States: Evidence for Thermal Activation and Deactivation through a Conical Intersection. Angew. Chem. Int. Ed. Engl. 1998, 37, 98-101, DOI: 10.1002/(SICI)15213773(19980202)37:1/2<98::AIDANIE98>3.0.CO;2-5.

(10) Wagner, P. J. In CRC Handbook of Organic Photochemistry and Photobiology, 1st ed.; Horspool, W., Song, P.-S., Eds.; CRC Press: New York, 1995; Chapter 38, pp 449-470.

(11) Yang, N. C.; Norrish, R. G. W. Cyclobutanol formation from irradiation of ketones. J. Am. Chem. Soc. 1958, 80, 2913-2914, DOI: 10.1021/ja01544a092.

(12) Yang, N. C.; Elliot, S. P.; Kim, B. The Mechanism of Photochemistry of Alkanones with $\gamma$ Hydrogens. J. Am. Chem. Soc. 1969, 91, 7551-7553, DOI: 10.1021/ja01054a079.

(13) Wagner, P. J.; Truman, R. J.; Scaiano, J. C. Substituent effects on hydrogen abstraction by phenyl ketone triplets. J. Am. Chem. Soc. 1985, 10\%, 7093-7096, DOI: $10.1021 / \mathrm{ja} 00310 \mathrm{a} 056$.

(14) Wagner, P. J. Solvent Effects on Type II Photoelimination of Phenyl Ketones. J. Am. Chem. Soc. 1967, 89, 5898-5901, DOI: 10.1021/ja00999a028.

(15) Weiss, R. G. In CRC Handbook of Organic Photochemistry and Photobiology, 1st ed.; Horspool, W., Song, P.-S., Eds.; CRC Press: New York, 1995; Chapter 39, pp 471-483.

(16) Wagner, P. J. Type II Photoelimination and Photocyclization of Ketones. Acc. Chem. Res. 1971, 4, 168-177, DOI: 10.1021/ar50041a002.

(17) Dinda, B. Essentials of Pericyclic and Photochemical Reactions; Springer International Publishing: Cham, 2017; pp 241-275, DOI: 10.1007/978-3-319-45934-9_8.

(18) Rubin, M. B. In CRC Handbook of Organic Photochemistry and Photobiology, 1st ed.; Horspool, W., Song, P.-S., Eds.; CRC Press: New York, 1995; Chapter 37, pp 437-448. 
(19) Wessig, P.; Mühling, O. Photochemical synthesis of highly functionalized cyclopropyl ketones. Helv. Chim. Acta 2003, 86, 865-893, DOI: $10.1002 /$ hlca.200390086.

(20) Wagner, P. J.; Chen, C.-P. Enhanced Photocyclization of alpha-Fluoro Ketones. $J$. Am. Chem. Soc. 1976, 98, 241-243, DOI: 10.1021/ja00417a043.

(21) Van Der Weerdt, A. J. A.; Cerfortain, H. Photochemistry of $\beta, \gamma$-unsaturated ketones. Tetrahedron Lett. 1981, 37, 2121-2130, DOI: 10.1016/S0040-4020(01)97968-2.

(22) Descotes, G. In CRC Handbook of Organic Photochemistry and Photobiology, 1st ed.; Hoorspool, W., Song, P.-S., Eds.; CRC Press: New York, 1995; Chapter 41, pp 501-512.

(23) Gudmundsdottir, A. D.; Lewis, T. J.; Randall, L. H.; Scheffer, J. R.; Rettig, S. J.; Trotter, J.; Wu, C. H. Geometric requirements for hydrogen abstractability and 1,4biradical reactivity in the Norrish/Yang type II reaction: Studies based on the solid state photochemistry and X-ray crystallography of medium-sized ring and macrocyclic diketones. J. Am. Chem. Soc. 1996, 118, 6167-6184, DOI: $10.1021 /$ ja953420a.

(24) Zepp, R. G.; Gumz, M. M.; Miller, W. L.; Gao, H. Photoreaction of valerophenone in aqueous solution. J. Phys. Chem. A 1998, 102, 5716-5723, DOI: 10.1021/jp9811301.

(25) Wagner, P. J. 1,5-Biradicals and FiveMembered Rings Generated by $\delta$-Hydrogen Abstraction in Photoexcited Ketones. Acc. Chem. Res. 1989, 22, 83-91, DOI: 10.1021/ar00159a001.

(26) Shaw, M. F. Photochemical Formation of Enols from Carbonyls. Ph.D. thesis, The University of Sydney, 2017.

(27) Seco, R.; Peñuelas, J.; Filella, I. Shortchain oxygenated VOCs: Emission and uptake by plants and atmospheric sources, sinks, and concentrations. Atmos. Environ. 2007, 41, 2477-2499, DOI: 10.1016/j.atmosenv.2006.11.029.

(28) Goldstein, A. H.; Galbally, I. E. Known and unexplored organic constituents in the earth's atmosphere. Environ. Sci. Technol. 2007, 41, 1514-1521, DOI: 10.1021/es072476p.

(29) Zhu, L.; Tang, Y.; Chen, Y.; Cronin, T. Wavelength-dependent photolysis of C3C7 aldehydes in the $280-330 \mathrm{~nm}$ region. Spectrosc. Lett. 2009, 42, 467-478, DOI: $10.1080 / 00387010903267195$.

(30) Jenkin, M. E.; Saunders, S. M.; Pilling, M. J. The tropospheric degradation of volatile organic compounds: A protocol for mechanism development. Atmos. Environ. 1997, 31, 81104, DOI: 10.1016/S1352-2310(96)00105-7.

(31) Rickard, A.; Young, J. The Master Chemical Mechanism (MCM) v3.2. http://mcm. leeds . ac.uk/MCM.

(32) Kharazmi, A. Investigating the Complex Photochemistry of Atmospheric Carbonyls. Ph.D. thesis, The University of New South Wales, 2019 .

(33) Coulson, R. D.; Yang, N. C. Deuterium Isotope Effects in the Photochemistry of 2Hexanone. J. Am. Chem. Soc. 1966, 88, 45114513.

(34) Feyter, S. D.; Diau, E. W.; Zewail, A. H. Femtosecond Dynamics of Norrish Type-II Reactions: Nonconcerted HydrogenTransfer and Diradical Intermediacy. Angew. Chem. Int. Ed. Engl. 2000, 39, 260-263, DOI: 10.1002/(SICI)15213773(20000103)39:1<260::AIDANIE260>3.0.CO;2-R.

(35) Encina, M. V.; Lissi, E. A. Photochemistry of alkyl ketones bearing $\gamma$-hydrogens. J. Photochem. 1976, 6, 173-181, DOI: 10.1016/0047-2670(76)85062-9.

(36) Nau, W. M. Pathways for the Photochemical Hydrogen Abstraction by $n, \pi^{*}$-Excited States. Ber. Bunsenges. Phys. Chem. 1998, 102, 476-485, DOI: 10.1002/bbpc.19981020329.

(37) Kawamura, K.; Steinberg, S.; Kaplan, I. R. Homologous series of C1-C10 monocarboxylic acids and C1-C6 carbonyls in Los Angeles air and motor vehicle exhausts. Atmos. Environ. 2000, 34, 4175-4191, DOI: 10.1016/S13522310(00)00212-0. 
(38) Atkinson, R.; Arey, J. Atmospheric Degradation of Volatile Organic Compounds. Chem. Rev. 2003, 103, 4605-4638, DOI: $10.1021 / \mathrm{cr} 0206420$.

(39) Atkinson, R.; Baulch, D.; Cox, Evaluated kinetic and photochemical data for atmospheric chemistry: Volume II - gas phase reactions of organic species. Atmos. Chem. Phys. 2006, 6, 3625-4055, DOI: https://doi.org/10.5194/acp-6-3625-2006.

(40) Wayne, R. P. Chemistry of Atmospheres, 3rd ed.; Oxford University Press: New York, 2006 .

(41) O'Connor, M. P.; Wenger, J. C.; Mellouki, A.; Wirtz, K.; Muñoz, A. The atmospheric photolysis of E-2-hexenal, Z-3-hexenal and E,E2,4-hexadienal. Phys. Chem. Chem. Phys. 2006, 8, 5236-5246, DOI: 10.1039/b611344c.

(42) Shen, L.; Fang, W. H. The reactivity of the 1,4-biradical formed by norrish type reactions of aqueous valerophenone: A QM/MM-based FEP study. J. Org. Chem. 2011, 76, 773-779, DOI: $10.1021 /$ jo101785z.

(43) Shemesh, D.; Lan, Z.; Gerber, R. B. Dynamics of triplet-state photochemistry of pentanal: Mechanisms of Norrish I, Norrish II, and $\mathrm{H}$ abstraction reactions. J. Phys. Chem. A 2013, 117, 11711-11724, DOI: 10.1021/jp401309b.

(44) Shemesh, D.; Blair, S. L.; Nizkorodov, S. A.; Gerber, R. B. Photochemistry of aldehyde clusters: cross-molecular versus unimolecular reaction dynamics. Phys. Chem. Chem. Phys. 2014, 16, 23861-23868, DOI: 10.1039/c4cp03130j.

(45) Dewar, M. J.; Doubleday, C. A MINDO/3 Study of the Norrish Type II Reaction of Butanal. J. Am. Chem. Soc. 1978, 100, 49354941, DOI: 10.1021/ja00484a002.

(46) Dorigo, A. E.; McCarrick, M. A.; Loncharich, R. J.; Houk, K. N. Transition structures for hydrogen atom transfers to oxygen. Comparisons of intermolecular and intramolecular processes and open- and closedshell systems. J. Am. Chem. Soc. 1990, 112, 7508, DOI: $10.1021 / \mathrm{ja00177a009.}$
(47) Sengupta, D.; Chandra, A. K. Studies on the Norrish type II reactions of aliphatic $\alpha$ diketones and the accompanying cyclization and disproportionation of 1:4 biradicals. $J$. Photochem. Photobiol. A 1993, 75, 151-162, DOI: 10.1016/1010-6030(93)80197-H.

(48) He, H. Y.; Fang, W. H.; Phillips, D. L. Photochemistry of butyrophenone: Combined complete-active-space self-consistent field and density functional theory study of norrish type I and II reactions. $J$. Phys. Chem. A 2004, 108, 5386-5392, DOI: 10.1021/jp037735l.

(49) Rowell, K. N.; Kable, S. H.; Jordan, M. J. T. Structural Effects on the Norrish Type I $\alpha$-Bond Cleavage of Tropospherically Important Carbonyls. The Journal of Physical Chemistry A 2019, 123, 10381-10396, DOI: 10.1021/acs.jpca.9b05534.

(50) Chen, X. B.; Fang, W. H. Norrish I vs II reactions of butanal: A combined CASSCF, DFT and MP2 study. Chem. Phys. Lett. 2002, 361, 473-482, DOI: 10.1016/S0009-2614(02)009570 .

(51) Marchetti, B.; Karsili, T. N. V.; Ashfold, M. N. R. Exploring Norrish type I and type II reactions: an ab initio mechanistic study highlighting singlet-state mediated chemistry. Phys. Chem. Chem. Phys. 2019, 27-30, DOI: $10.1039 /$ c8cp07292b.

(52) Cronin, T. J.; Zhu, L. Dye Laser Photolysis of n-Pentanal from 280 to $330 \mathrm{~nm}$. The $J$. Phys. Chem. A 1998, 102, 10274-10279, DOI: 10.1021/jp982487s.

(53) Granovsky, A. A. Extended multiconfiguration quasi-degenerate perturbation theory: The new approach to multi-state multi-reference perturbation theory. $J$. Chem. Phys. 2011, 134, 214113, DOI: 10.1063/1.3596699.

(54) Kletskii, M. E.; Lisovin, A. V.; Burov, O. N.; Kurbatov, S. V. Competing mechanisms of Norrish and Norrish-like reactions in a wide range of systems - From carbonyl compounds to nitrogen oxide donators. Comp. Theo. Chem. 2014, 1047, 55-66, DOI: 10.1016/j.comptc.2014.08.025. 
(55) Köhn, A.; Tajti, A. Can coupled-cluster theory treat conical intersections? J. Chem. Phys. 2007, 127, 044105, DOI: $10.1063 / 1.2755681$.

(56) Budzak, S.; Scalmani, G.; Jacquemin, D. Accurate Excited-State Geometries: A CASPT2 and Coupled-Cluster Reference Database for Small Molecules. J. Chem. Theory Comp. 2017, 13, 6237-6252, DOI: 10.1021/acs.jctc.7b00921.

(57) Rowell, K.; Kable, S.; Jordan, M. J. T. Predicting Carbonyl Excitation Energies Efficiently Using EOM-CC Trends. 2020, DOI: 10.26434/chemrxiv.12917369.v1.

(58) Levine, B. G.; Ko, C.; Quenneville, J.; Martínez, T. J. Conical intersections and double excitations in timedependent density functional theory. Mol. Phys. 2006, 104, 1039-1051, DOI: $10.1080 / 00268970500417762$.

(59) Jursic, B. S. Exploring the potential energy surface of the hydrogen abstraction reaction from hydrochloric acid with hydrogen and methyl radicals using ab initio and density functional theory methods. An example of a polar radical hydrogen abstraction reaction. Chem. Phys. Lett. 1997, 264, 113-119, DOI: 10.1016/S0009-2614(96)01317-6.

(60) Chan, B.; Gilbert, A. T.; Gill, P. M.; Radom, L. Performance of density functional theory procedures for the calculation of proton-exchange barriers: Unusual behavior of M06-type functionals. J. Chem. Theory Comp. 2014, 10, 3777-3783, DOI: $10.1021 / \mathrm{ct} 500506 \mathrm{t}$.

(61) Szori, M.; Fittschen, C.; Csizmadia, I. G.; Viskolcz, B. Allylic H-abstraction mechanism: The potential energy surface of the reaction of propene with $\mathrm{OH}$ radical. J. Chem. Theory Comp. 2006, 2, 1575-1586, DOI: 10.1021/ct600140b.

(62) Izsák, R.; Szori, M.; Knowles, P. J.; Viskolcz, B. High accuracy ab initio calculations on reactions of $\mathrm{OH}$ with 1alkenes. The case of propene. J. Chem. Theory Comp. 2009, 5, 2313-2321, DOI: $10.1021 /$ ct900133v.
(63) Andersson, M. P.; Uvdal, P. New scale factors for harmonic vibrational frequencies using the B3LYP density functional method with the triple- $\zeta$ basis Set $6-311+\mathrm{G}(\mathrm{d}, \mathrm{p}) . J$. Phys. Chem. A 2005, 109, 2937-2941, DOI: 10.1021/jp045733a.

(64) Alecu, I. M.; Zheng, J.; Zhao, Y.; Truhlar, D. G. Computational thermochemistry: Scale factor databases and scale factors for vibrational frequencies obtained from electronic model chemistries. J. Chem. Theory Comp. 2010, 6, 2872-2887, DOI: $10.1021 /$ ct100326h.

(65) Liu, J.; Liang, W. Analytical approach for the excited-state Hessian in time-dependent density functional theory: Formalism, implementation, and performance. J. Chem. Phys. 2011, 135, 184111, DOI: 10.1063/1.3659312.

(66) Furche, F.; Ahlrichs, R. Adiabatic timedependent density functional methods for excited state properties. The J. Chem. Phys. 2002, 117, 7433-7447, DOI: $10.1063 / 1.1508368$.

(67) Chattopadhyay, S.; Chaudhuri, R. K.; Mahapatra, U. S. Ab initio multireference investigation of disjoint diradicals: Singlet versus triplet ground states. ChemPhysChem 2011, 12, 2791-2797, DOI: $10.1002 /$ cphc. 201100430.

(68) Yang, K. R.; Jalan, A.; Green, W. H.; Truhlar, D. G. Which Ab initio wave function methods are adequate for quantitative calculations of the energies of biradicals? the performance of coupled-cluster and multireference methods along a single-bond dissociation coordinate. J. Chem. Theory Comp. 2013, 9, 418-431, DOI: 10.1021/ct3009528.

(69) Ess, D. H.; Cook, T. C. Unrestricted prescriptions for open-shell singlet diradicals: Using economical Ab initio and density functional theory to calculate singlettriplet gaps and bond dissociation curves. $J$. Phys. Chem. A 2012, 116, 4922-4929, DOI: 10.1021/jp300633j.

(70) López-Carballeira, D.; Ruipérez, F. Evaluation of modern DFT functionals and G3nRAD composite methods in the modelization 
of organic singlet diradicals. J. Mole. Model. 2016, 22, 76, DOI: 10.1007/s00894-016-2950$\mathrm{Z}$.

(71) Fang, W. H. A CASSCF study on photodissociation of acrolein in the gas phase. $J$. Am. Chem. Soc. 1999, 121, 8376-8384, DOI: 10.1021/ja982334i.

(72) Gozem, S.; Melaccio, F.; Valentini, A.; Filatov, M.; Huix-Rotllant, M.; Ferré, N.; Frutos, L. M.; Angeli, C.; Krylov, A. I.; Granovsky, A. A. et al. Shape of multireference, equation-of-motion coupled-cluster, and density functional theory potential energy surfaces at a conical intersection. J. Chem. Theory Comp. 2014, 10, 3074-3084, DOI: $10.1021 /$ ct500154k.

(73) Barca, G. M.; Gilbert, A. T.; Gill, P. M. Simple Models for Difficult Electronic Excitations. J. Chem. Theory Comp. 2018, 14, 1501-1509, DOI: 10.1021/acs.jctc.7b00994.

(74) Huix-Rotllant, M.; Nikiforov, A.; Thiel, W.; Filatov, M. In Density-Functional Methods for Excited States; Ferré, N., Filatov, M., Huix-Rotllant, M., Eds.; Springer International Publishing: Zurich, 2015; Chapter 11, pp 445-476, DOI: 10.1007/128_2015_63.

(75) Arnett, J. F.; Newkome, G.; Mattice, W. L.; McGlynn, S. P. Excited Electronic States of the $\alpha$-Dicarbonyls. J. Am. Chem. Soc. 1974, 96, 4385-4392, DOI: 10.1021/ja00821a007.

(76) Dykstra, C. E.; Schaefer, H. F. Electronic Structure of Dicarbonyls. Glyoxal Excited States. J. Am. Chem. Soc. 1976, 98, 401406, DOI: 10.1021/ja00418a014.

(77) Jacquemin, D. What is the Key for Accurate Absorption and Emission Calculations, Energy or Geometry? J. Chem. Theory Comp. 2018, 14, 1534-1543, DOI: 10.1021/acs.jctc.7b01224.

(78) Lee, T. J.; Taylor, P. R. A diagnostic for determininig the quality of single-reference electron correlation methods. Int. J. Quantum Chem. 1989, 23, 199-207.

(79) Lee, T. J. Comparison of the $\mathcal{T}_{1}$ and $\mathcal{D}_{1}$ diagnostics for electronic structure theory: A new definition for the open-shell $\mathcal{D}_{1}$ diagnostic.
Chem. Phys. Lett. 2003, 372, 362-367, DOI: 10.1016/S0009-2614(03)00435-4.

(80) Page, C. S.; Olivucci, M. Ground and excited state CASPT2 geometry optimizations of small organic molecules. Journal of Computational Chemistry 2003, 24, 298-309, DOI: 10.1002/jcc. 10145 .

(81) Huix-Rotllant, M.; Ferré, N. Triplet state photochemistry and the three-state crossing of acetophenone within time-dependent densityfunctional theory. J. Chem. Phys. 2014, 140, DOI: $10.1063 / 1.4869802$.

(82) Teh, H. H.; Subotnik, J. E. The Simplest Possible Approach for Simulating $S_{0}-S_{1}$ Conical Intersections with DFT/TDDFT: Adding One Doubly Excited Configuration. J. Phys. Chem. Lett. 2019, 10, 3426-3432, DOI: 10.1021/acs.jpclett.9b00981.

(83) Keller-Rudek, G. K., Hannelore Moortgat; Sander, R.; Sörensen, R. The MPIMainz UV/VIS Spectral Atlas of Gaseous Molecules of Atmospheric Interest. 2019; www . uv-vis-spectral-atlas-mainz org.

(84) Shu, J.; Peterka, D. S.; Leone, S. R.; Ahmed, M. Tunable synchrotron vacuum ultraviolet ionization, time-of-flight investigation of the photodissociation of trans-crotonaldehyde at $193 \mathrm{~nm}$. J. Phys. Chem. A 2004, 108, 7895-7902, DOI: 10.1021/jp049638d.

(85) Johnstone, D. E.; Sodeau, J. R. Photochemistry of the matrix-isolated $\alpha, \beta-$ unsaturated aldehydes acrolein, methacrolein and crotonaldehyde at $4.2 \mathrm{~K}$. J. Chem. Soc. Faraday Trans. 1992, 88, 409-415, DOI: 10.1039/FT9928800409.

(86) Allen, E. R.; Pitts, J. N. The Vapor-Phase Photolysis of trans-Crotonaldehyde. J. Am. Chem. Soc. 1968, 91, 3135-3139, DOI: 10.1021/ja01040a005.

(87) Blacet, F. E.; Lu Valle, J. E. The Photolysis of the Aliphatic Aldehydes. VII. Crotonaldehyde at Elevated Temperatures. J. Am. Chem. Soc. 1939, 61, 273-276, DOI: 10.1021/ja01871a015. 
(88) Coomber, J. W.; Pitts, J. N.; Schrock, R. R. The detection of ethylketene and enolcrotonaldehyde in the vapour-phase Photolysis of trans-crotonaldehyde. ChemComm 1968, 190-191, DOI: 10.1039/C1968000190B.

(89) McDowell, C. A.; Sifniades, S. Isomerization as a Primary Process in the Photolysis of Crotonaldehyde. J. Am. Chem. Soc. 1962, 84, 4606-4607, DOI: 10.1021/ja00882a057.

(90) Houk, K. N. The Frontier Molecular Orbital Theory of Cycloaddition Reactions. Acc. Chem. Res. 1975, 8, 361-369, DOI: 10.1021/ar50095a001.

(91) Kumar, S.; Kumar, V.; Singh, S. Pericyclic Reactions; 2016; Chapter 3, pp 77-144, DOI: 10.1016/B978-0-12-803640-2.00003-8.

(92) McLafferty, F. W. Mass Spectrometric Analysis: Molecular Rearrangements. Anal. Chem. 1959, 31, 82-87, DOI: 10.1021/ac60145a015.

(93) Wagner, P. J. Misleading sigmas and neglected geometries: the effects of methyl substitution on rates of triplet state hydrogen abstraction by the benzoyl group. Pure Appl. Chem. 1977, 49, 259-270, DOI: 10.1351/pac197749030259.

(94) Lewis, F. D.; Hilliard, T. A. Photochemistry of Methyl-Substituted Butyrophenones. The Nature of the 1,4-Biradical Intermediates. $J$. Am. Chem. Soc. 1970, 92, 6672-6674, DOI: 10.1021/ja00725a066.

(95) Anslyn, E. V.; Dougherty, D. A. Modern Physical Organic Chemistry; University Science Books: Sausalito, CA, 2006.

(96) Donahue, N. M. Reaction Barriers: Origin and Evolution. Chem. Rev. 2003, 103, 45934604, DOI: $10.1021 / \mathrm{cr} 020650 \mathrm{~g}$.

(97) Shiozaki, T. BAGEL: Brilliantly Advanced General Electronic-structure Library. WIREs Computational Molecular Science 2018, 8, e1331, DOI: 10.1002/wcms.1331.

(98) Bonn, B.; Magh, R.-K.; Rombach, J.; Kreuzwieser, J. Biogenic isoprenoid emissions under drought stress: Different responses for isoprene and terpenes. Biogeosciences Discuss. 2019, 1-30, DOI: 10.5194/bg-2019-227.
(99) Harvard Atmospheric Chemistry Modeling Group, GEOS-Chem 12.4.0. http://acmg. seas.harvard.edu/geos/index.html.

(100) Jenkin, M. E.; Saunders, S. M.; Derwent, R. G.; Pilling, M. J. Development of a reduced speciated VOC degradation mechanism for use in ozone models. Atmos. Environ. 2002, 36, 4725-4734, DOI: 10.1016/S13522310(02)00563-0.

(101) Chen, Y.; Zhu, L.; Francisco, J. S. Wavelength-Dependent Photolysis of nButyraldehyde and i-Butyraldehyde in the 280-330 nm Region. J. Phys. Chem. A 2002, 7755-7763, DOI: 10.1021/JP014544E.

(102) Wagner, P. J.; Hammond, G. S. Mechanism of Type II Photoelimination. J. Am. Chem. Soc. 1965, 87, 4009-4011, DOI: 10.1021/ja01095a059. 